\title{
双-1,2,3-三唑化合物的合成与应用研究进展
}

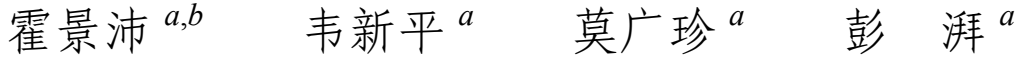 \\ 钟铭丽 $a$ 陈任宏 ${ }^{c}$ 汪朝阳*,a \\ ( ${ }^{a}$ 华南师范大学化学与环境学院 教育部环境理论化学重点实验室 广州 510006) \\ ( $b$ 华南理工大学化工学院 广州 510630) \\ ( ${ }^{c}$ 广东食品药品职业学院 广州 510520)
}

\begin{abstract}
摘要 双-1,2,3-三唑化合物作为重要的 $\mathrm{N}$-杂环化合物, 近年来吸引了越来越多不同领域化学家的关注. 为了合成对称 的与不对称的双三坐，一些合成中的新发现、新策略、新技术不断涌现. 从所用原料与合成方式等角度，可分为 Click 反应-氧化偶联法、原料组合法、浓度控制法、基团活性差异法、基团保护法、控制投料比法、特殊试剂法等.同时，介 绍了相关双三唑化合物在药物化学、配位化学、化学传感器、分子识别、生物化学、超分子化学、表面活性剂等应用 领域的新进展，并对双三唑化合物的合成与应用前景进行了展望.
\end{abstract}

关键词 双-1,2,3-三唑; 合成; Click 反应; 叠氮化物; 炔烃; 进展

\section{Progresses in Syntheses and Applications of Bis-1,2,3-triazoles}

\author{
Huo, Jingpei ${ }^{a, b}$ \\ Wei, Xinping $^{a}$ \\ Mo, Guangzhen ${ }^{a}$ \\ Peng, $\mathrm{Pai}^{a}$ \\ Zhong, Mingli ${ }^{a}$ \\ Chen, Renhong ${ }^{c}$ \\ Wang, Zhaoyang*,a \\ $\left({ }^{a}\right.$ Key Laboratory of Theoretical Chemistry of Environment, Ministry of Education, School of Chemistry and \\ Environment, South China Normal University, Guangzhou 510006) \\ $\left({ }^{b}\right.$ School of Chemistry and Chemical Engineering, South China University of Technology, Guangzhou 510630) \\ ( ${ }^{c}$ Guangdong Food and Drug Vocational College, Guangzhou 510520)
}

\begin{abstract}
As a kind of important N-heterocyclic compounds, bis-1,2,3-triazoles have drawn more and more interest of chemists in different research territories. In order to synthesize symmetric and unsymmetrical bis-1,2,3-triazoles, many synthetic strategies, methods and techniques, such as click reaction-oxidative coupling, the combination of starting materials, the concentration control method, the utilization of different active groups, the protection technique, the control of feed ratio, the use of special reagents, and so on, have been developed recently. Classified as different substrates and synthetic approaches, these research progresses are summarized. At the same time, the new progresses in the applications of bis-1,2,3-triazoles in different fields, including medicinal chemistry, coordination chemistry, chemical sensor, molecular recognition, biochemistry, supramolecular chemistry and surfactant are also mentioned in this review. It is pointed out that how to cleverly design and efficiently synthesize the novel bis-1,2,3-triazoles for some special purpose via the simple general methods is still a great challenge.

Keywords bis-1,2,3-triazoles; synthesis; click reaction; azides; alkynes; progress
\end{abstract}

作为一种重要的 $\mathrm{N}$-杂环化合物, 1,2,3-三唑化合物 因其独特的功能性为人们所重视, 特别是简洁的 $\mathrm{Cu}(\mathrm{I})$ 催化 Click 反应(CuAAC)使 1,2,3-三唑的分子设计与合 成倍受关注 ${ }^{[1]}$. 近年来, 随着 CuAAC 研究的不断深入,
各种双-1,2,3-三唑化合物不仅在药物设计中日益受到瞩

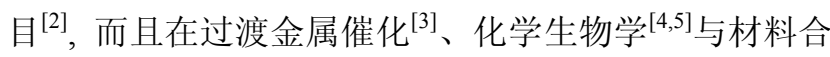
成 ${ }^{[6-8]}$ 等领域发挥了重要的作用, 但以往的综述报道仅 仅是分别从不同的侧面，如铜催化剂 ${ }^{[1]}$ 、功能性反应底

\footnotetext{
*E-mail: wangzy@scnu.edu.cn

Received July 15, 2013; revised August 30, 2013; published online September 6, 2013.
}

Project supported by the National Natural Science Foundation of China (No. 20772035), the 3rd Talents Special Funds of Guangdong Higher Education (No. Guangdong-Finance-Education [2011]431) and the Natural Science Foundation of Guangdong Province (No. S2011010001556).

国家自然科学基金(No. 20772035)、广东省高等学校人才引进专项资金(No. 粤财教[2011]431 号)、广东省自然科学基金(No. S2011010001556)资助项 目. 
物 ${ }^{[9]}$ 、新型材料合成 ${ }^{[7,8]}$ 等偶尔提及双-1,2,3-三唑化合物, 却没有对其进行系统的综述. 鉴于此, 本文按照不同的 合成方式, 对各种对称与不对称的双-1,2,3-三唑近年来 合成与应用的新进展进行了综述, 希望能够为更多新型 双-1,2,3-三唑类化合物目标分子的设计与合成提供借 鉴.

\section{1 对称双-1,2,3-三唑化合物的合成}

对称的双-1,2,3-三唑化合物, 即得到化合物分子是 对称的. 从所用的原料类型来看, 有四种不同的组合方 式得到对称双三唑, 但在各种原料的组合中也涉及到一 些不同的合成方法.

\section{1 基于一叠氮化物与一炔烃的合成}

早在 2002 年, Sharpless 等 ${ }^{[10]}$ 在合成单三唑化合物的 过程中, 就意外地在反应体系中发现了双三唑化合物的 存在, 但将其当成了副产物, 并没有对其进行详细的探 讨. 直到 2007 年, Burgess 等 ${ }^{[11]}$ 对此意外现象进行了研 究, 发现碱的种类在单三唑偶联成双三唑的过程中至关 重要, 其中碳酸钠对双三唑的形成最为有利(Eq. 1). 后 来, Jeon 等 ${ }^{[12]}$ 一些实验结果也证明了这一点.

除碱是重要的因素外, 温度和催化剂也有一定的影 响. 2011 年, Cuevas-Yanez 等 ${ }^{[13]}$ 以茮基叠氮化物和不同 的炔烃为原料, 以 $\mathrm{CuI}$ 为催化剂, 发现在 $-35{ }^{\circ} \mathrm{C}$ 、高浓 度 $\mathrm{NaOH}$ 条件下, 在甲醇溶液中发生 $\mathrm{CuAAC}$-氧化二聚 反应生成对称的 5,5'-双三唑化合物; 而在甲醇回流温度
下反应时，生成的双三唑化合物会跟 $\mathrm{CuI}-\mathrm{NaOH}$ 催化体 系发生反应，自身消耗而生成单三唑化合物. 因此，控 制好温度对反应的有效进行起着至关重要的作用 ${ }^{[13]}$. 但是，相比之下，温度对双三唑化合物形成的影响，不 及不同碱种类的影响那么重要(Eq. 2) ${ }^{[12]}$.

2012 年 Vidal 课题组 ${ }^{[14]}$ 发现, 对于糖基叠氮化物与 苯乙炔的反应, 使用 $\mathrm{CuCl}$ 催化时产物以双三唑为主, 但 $\mathrm{CuI}, \mathrm{CuBr}$ 作催化剂时却主要生成 5-甾代的单三唑. Jeon 等 ${ }^{[12]}$ 研究了烷基叠氮化物和丙炔酰胺类化合物的 Click 反应，发现以 $\mathrm{CuI}, \mathrm{CuBr}$ 作催化剂时, 生成单三唑 的同时也得到大量的双三唑化合物 (二者总产率通常 $90 \%$ 以上), 但使用硫酸铜/抗坏血酸钠催化体系则仅以 $94 \%$ 以上的产率生成单三唑化合物. 更为奇怪的是, 这 个规律也只适用于丙炔酰胺类底物, 对于丙炔酸、丙炔 酸酯却不会出现类似的情况 ${ }^{[12]}$.

基于一叠氮化物与一炔烃合成对称的双三唑，除上 述的 Click 反应-氧化偶联法, 也可选择较为特殊的试剂 作为底物合成对称双三唑化合物. 例如, Rissanen 课题 组 ${ }^{[15]}$ 以多官能团的底物 4 先合成单三唑化合物, 再利用 其中继续存在的醛基和羟基两种基团分别与邻苯二胺、 醋酸锌进行反应, 形成含有双三唑结构的配合物 (Scheme 1), 该配合物在胶束的作用下对水中磷酸根有 很强的键合作用. 雷鸣等 ${ }^{[16]}$ 以化合物 $\mathbf{5}$ 为原料, 利用水 中的三组分一锅煮反应，在不需要碱的情况下，基于一 叠氮化物与一炔烃而也合成了对称的双三唑(Eq. 3).
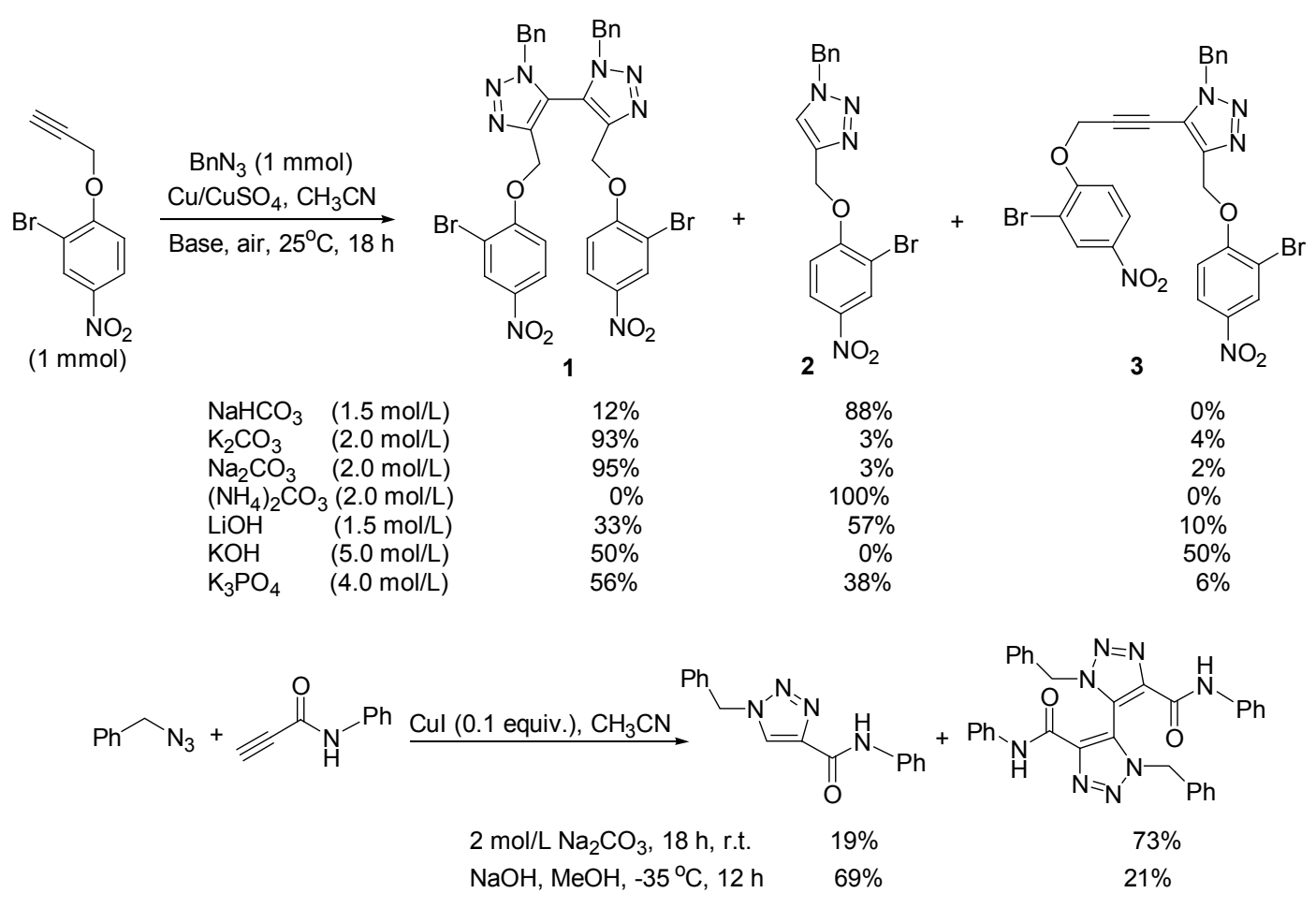


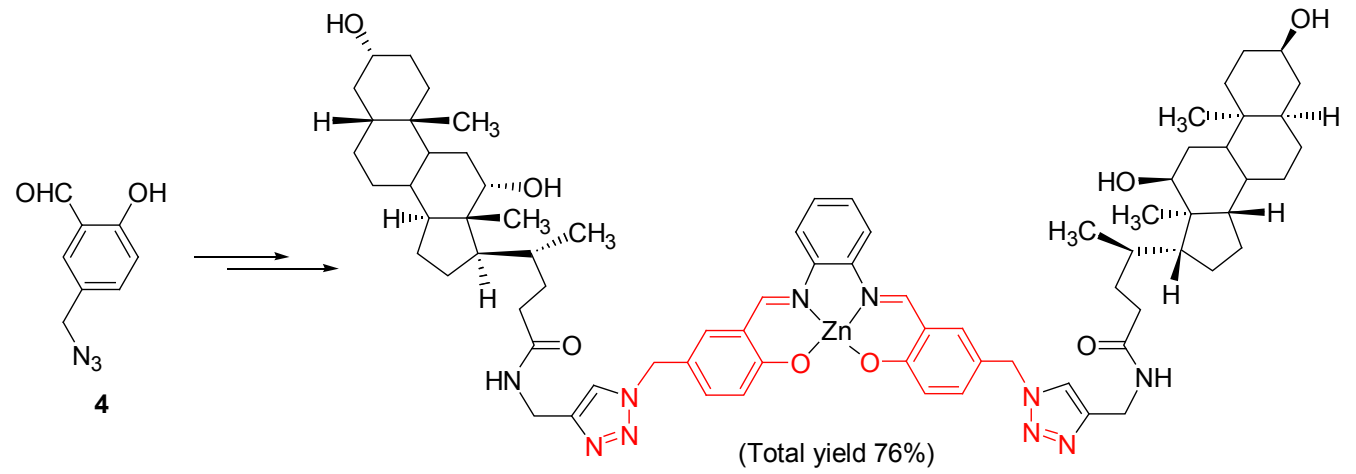

Scheme 1

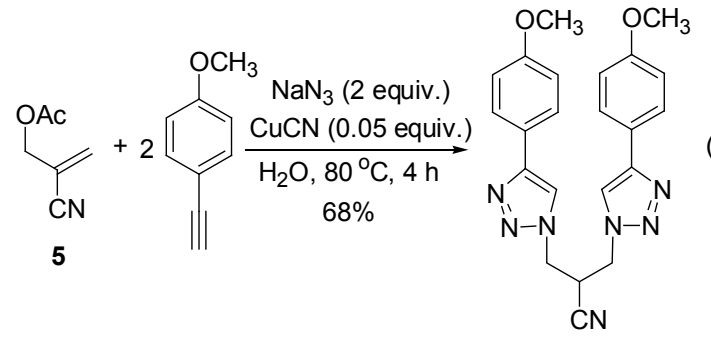

\section{2 基于二叠氮化物与一炔烃的合成}

如果用二卤代烃和叠氮化钠作为原料, 发生亲核取 代反应生成有机二叠氮化物中间体, 再进一步地与炔烃 发生双 Click 反应，也可得到对称的双三唑化合物. 例 如, Wang 等 ${ }^{[17]}$ 以 $\mathrm{CuI}$ 为催化剂, 采用三组分水相法合成 对称双三唑, 产物容易过滤分离, 而且不需要引入任何 还原剂和氧气保护. 反应中二卤代烃无论是邻位还是间 位, 都能以 $95 \%$ 以上的产率高选择性地合成 1,4-二取代 的双-1,2,3-三唑化合物(Eq. 4).<smiles></smiles>

除常见的以二卤代烃方式得到二叠氮化物外, 通过 一些特别的方式也可生成二叠氮化物而合成对称的双 三唑. 例如, 2010 年, Shreeve等 ${ }^{[18]}$ 在用五氟磺酰基烷烃、 叠氮化钠和末端炔合成三唑化合物时, 意外发现当温度 为 $60{ }^{\circ} \mathrm{C}$ 时仅生成单三唑化合物, 而温度升高到 $80{ }^{\circ} \mathrm{C}$ 时 有 $10 \%$ 的双三唑化合物生成一一原因是底物 6 中的 OTs 基团和 $\mathrm{SF}_{5}$ 基团同时被叠氮基取代，生成了二叠氮化物， 然后与末端炔烃发生双 Click 反应一一当末端炔烃的取 代基由丁基代替三氟甲基时，双三唑的产率可达到 60\% (Scheme 2).

许多对称的双三唑化合物是重要的药物 ${ }^{[19]}$. 例如, Nandurdikar 等 ${ }^{[20]}$ 以二叠氮化物 7 与炔类化合物 8 反应, 可以制备能够释放出 NO 而用于治疗癌症的药物. 但在

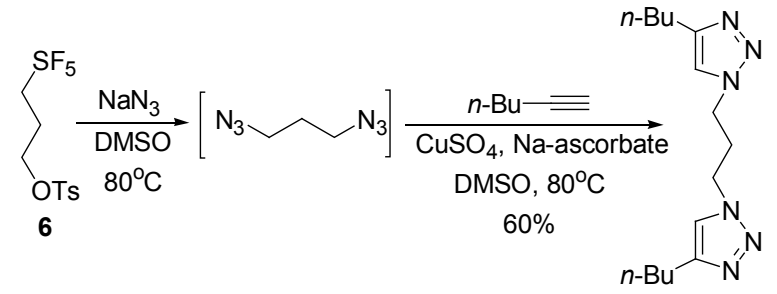

Scheme 2

合成中他们发现，当催化体系为硫酸铜/抗坏血酸钠时， 得到的产物以化合物 9(即正常的 Click 反应产物)为主; 而当催化体系为 $\mathrm{CuI} / N, N$-二异丙基乙胺(DIPEA)时，由 于 DIPEA 的位阻作用，主要发生 Click 反应-氧化偶联 反应，即以化合物 $\mathbf{1 0}$ 为主(Eq. 5).

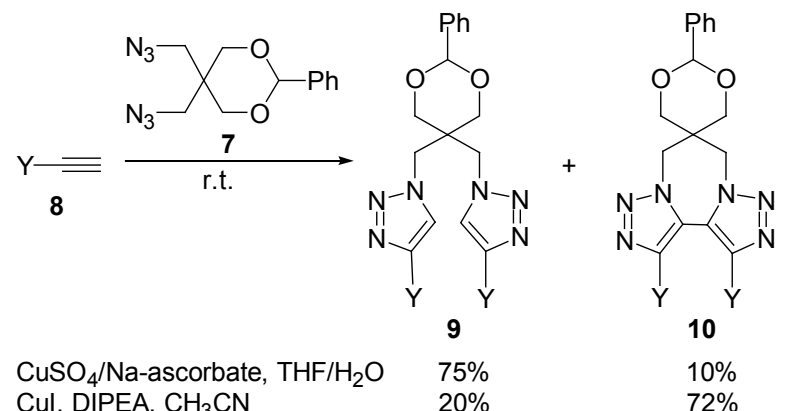
Cul, DIPEA, $\mathrm{CH}_{3} \mathrm{CN}$<smiles>[Y]C#C[N+](=O)[O-]</smiles>

2012 年, Gaur 等 ${ }^{[21]}$ 以多甲氧基取代的联苯二叠氮 化物 11(图 1)为底物与系列炔类化合物反应，合成的对 称双三唑具有显著的抗真菌活性. 类似地，以含有咪唑 基团的二叠氮化物 12(图 1)为底物，也可合成系列具有 抑菌性能的对称双三唑化合物 ${ }^{[22]}$. 最近, Desai 课题 组 ${ }^{[23]}$ 以烷基二叠氮化物 13 为底物, 合成了对称双三唑 14(图 1), 其被用于抗血栓药的篮选; 而 Drašar 课题 
组 ${ }^{[24]}$ 以吡啶环二叠氮化物 15 和甾类衍生物 16 为原料, 合成了用于甾类药物篎选的对称双三唑化合物.
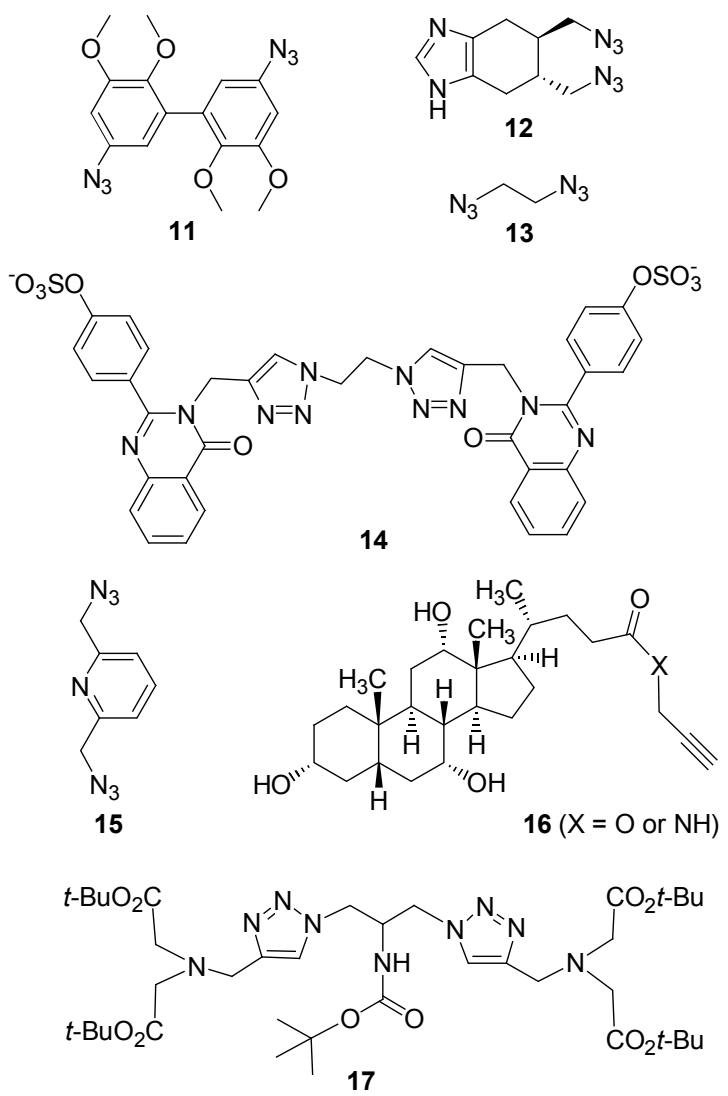

图 1 一些合成对称双三唑中的原料和目标分子

Figure 1 Some molecules in the symmetric synthesis of bis1,2,3-triazoles

对称的双三唑化合物除生物医药领域的应用之外, 还可应用于金属离子检测、化学传感器等多个领域. 例 如, Benoist 等 ${ }^{[25]}$ 利用 Click 反应合成了对称的双三唑化 合物 17(图 1), 由于同时引入了多个含 $\mathrm{O}$ 、含 $\mathrm{N}$ 官能团, 从而更加有利于与金属离子络合, 故该三唑化合物对过 渡金属离子有着较强的配合作用, 可用于放射性治疗和 场致发光等领域.

萠是一种苂光基团, 因此 Chung 课题组 ${ }^{[26]}$ 以含萠的 炔烃作为底物, 通过 Click 反应合成对称的双三坐化合 物(Eq. 6), 该化合物在乙腈溶剂中对 $\mathrm{Ni}^{2+}, \mathrm{Pb}^{2+}, \mathrm{Cd}^{2+}$, $\mathrm{Zn}^{2+}$ 等多种过渡金属离子具有双重模式的识别功能. 如 果由含多个 $\mathrm{OCH}_{2} \mathrm{CH}_{2}$ 链节的二叠氮化物 19(图 2) 来代替 二叠氮化物 18 进行合成, 由于产物中多个氧原子的存 在能使目标分子与金属离子的络合能力增强, 从而更加 有利于这些对称的双三唑化合物在极性更大的甲醇溶 剂中高选择性地检测 $\mathrm{Ag}^{+}, \mathrm{Hg}^{2+}$ 等金属离子 ${ }^{[27]}$.

蒽也是一种荧光基团, 而且糖基中的氧原子可为金 属离子的结合提供位点, 因此 $\mathrm{Wu}$ 课题组 ${ }^{[28]}$ 以葱基二叠
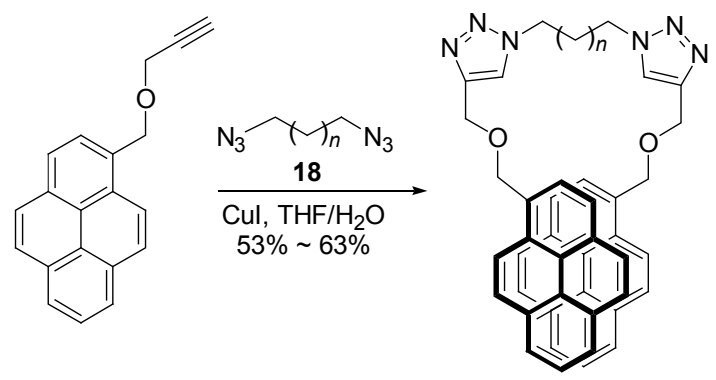

(6)

氮化物 20 和糖基炔化物 21(图 2)为原料, 通过 Click 反 应合成了新型对称双三唑类的荧光传感器, 为 $\mathrm{Hg}^{2+}$, $\mathrm{Cu}^{2+}, \mathrm{Pb}^{2+}, \mathrm{Ni}^{2+}$ 等重金属离子的苂光检测提供了一种新 的途径. 如果以氨基酸基炔化物与葱基二叠氮化物 20 进行 Click 反应, 得到的对称双三唑可作为蛋白质类生 物分子苂光标记的有机探针 ${ }^{[29]}$.

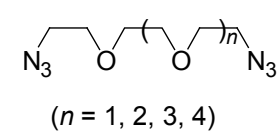

19

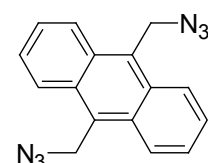

20<smiles>C#CCNCC1OC2OC(C)(C)OC1C2CC(C)=O</smiles>

21

图 2 用于合成苂光类对称双三唑的原料分子结构式 Figure 2 Some structures of starting materials for fluorescent symmetric bis-1,2,3-triazoles

菲也是一种苂光基团，二茂铁类化合物具有光电性 能, 因此最近 Molina 课题组 ${ }^{[30]}$ 以含二茂铁基二叠氮化 物 22 为原料, 成功地通过 Click 反应将菲引入了双三唑 (Scheme 3). 其实，利用含二茂铁基的炔化物 23 为原料， 也可得到以二茂铁-三唑为核心的对称双三唑化合物 (Scheme 3). 这些双三唑化合物最为显著的特点, 是其 氧化还原性能与光学性能, 都能使其成为一种对 $\mathrm{Hg}^{2+}$ 进行选择性识别的分子传感器.

基于二叠氮化物与一炔烃合成的双三唑，亦可应用 于超分子化学与分子机器等方面的研究. 例如黄飞鹤课 题组 ${ }^{[31]}$ 曾以二叠氮化物 24 合成了双三唑 25 (Eq. 7), 其 中三唑环的氮原子有助于氢键的相互作用, 从而有利于 化合物 25 与柱芳烃进一步在双三唑官能团之间的烷基 链处形成轮烷. Ol'shevskaya 等 ${ }^{[32]}$ 则以碳硼烷衍生的二 叠氮化物为原料, 通过 Click 反应合成了含碳硼烷结构 的对称双三唑化合物，这些化合物能与甲基碘反应得到 相应水溶性的三唑盐.

\section{3 基于二炔化物与一叠氮化物的合成}

基于二炔化物与一叠氮化物的 Click 反应，来合成 对称双三坐的报道, 在文献中也非常常见. 例如, Hsung 


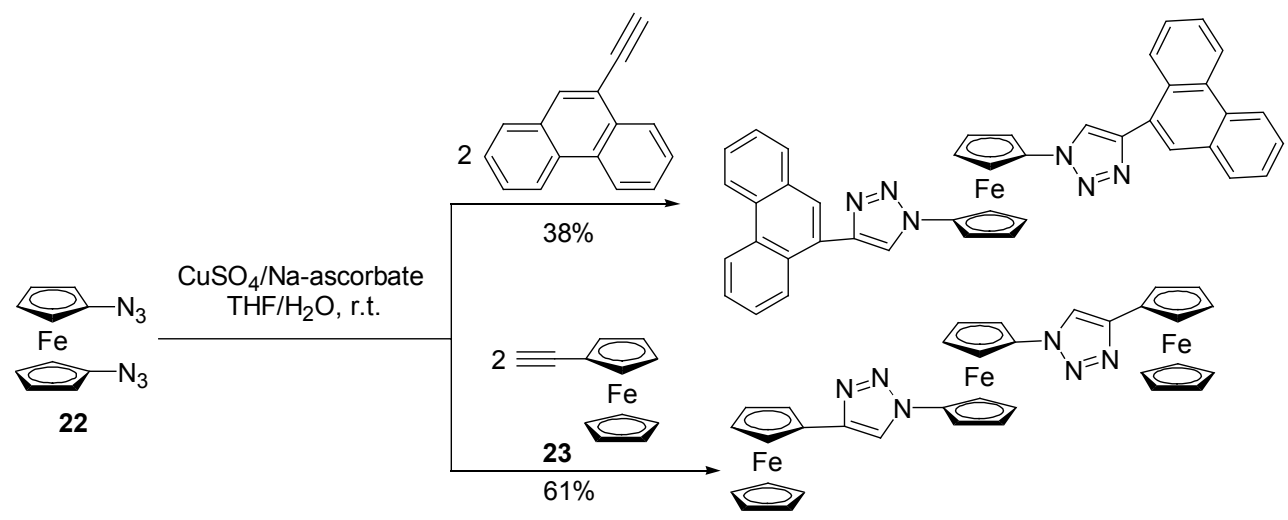

Scheme 3<smiles>OCc1ccc(O)cc1</smiles>

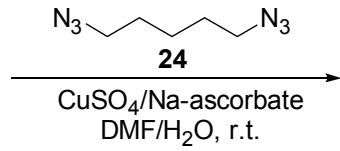

$60 \%$<smiles>OCc1ccc(OCc2cn(CCCCCn3cc(COc4ccc(CO)cc4)nn3)nn2)cc1</smiles>

课题组 ${ }^{[33]}$ 曾利用二炔化物 26(图 3) 为原料合成过对称的 双三唑化合物. 如果采用微波 $(\mathrm{MW})$ 技术, 可以使由二 炔化物 27(图 3)合成双三唑的反应时间由 $24 \mathrm{~h}$ 缩短到 12 $\min$ 而产率相当 ${ }^{[34]}$.

由 27 等二炔化物合成系列对称双三唑可作为配体, 它们能够与 $\mathrm{Pd}^{2+}$ 自组装形成配位饱和的四重螺旋笼状 配合物 $\left[\mathrm{Pd}_{2} \mathrm{~L}_{4}\right]\left(\mathrm{BF}_{4}\right)_{4}$, 其在固态和溶液中都能稳定存在, 可望应用于催化剂、分子识别和药物传输等领域 ${ }^{[35]}$. 由 二炔化物 28(图 3)合成的双三唑化合物，可用作对双齿 配体，其铑 ${ }^{[36]}$ 、铜 ${ }^{[37]}$ 等过渡金属离子具有很强的络合作 用. 甚至由 28 与叠氮二茂铁合成的双三唑 29 (图 3), 可 直接应用于 $\mathrm{Pb}^{2+}, \mathrm{Ni}^{2+}$ 等金属离子的光学识别 ${ }^{[30]}$. 钴配 合的夹心化合物 30(图 3), 能在空气和水分中高度稳定 地存在, 生成的双三唑化合物可望作为双齿配体应用于
催化领域 ${ }^{[38]}$.

吡啶中氮原子具有配位功能，因此将吡啶环引入双 三唑可扩大双三唑在配位化学中的应用范围. 例如, Flood 课题组 ${ }^{[39]}$ 以二炔化合物 31(图 3)进行 Click 反应, 所得的对称双三唑化合物是一种三齿配体，其对过渡金 属离子如 $\mathrm{Fe}^{2+}, \mathrm{Ru}^{2+}, \mathrm{Eu}^{3+}$ 有着非常稳定的络合作用. 为 避免常见的气态末端炔烃带来的称量不便, Fletcher 等 ${ }^{[00]}$ 引入三甲基硅基(TMS)来保护二炔化合物，并使用 被保护后为固态的二炔化合物 $\mathbf{2 8 , 3 2}$ (图 3)进行了合成 与应用研究.

四硫富瓦烯类含硫杂环本身良好的光电性能，使其 衍生的对称双三唑化合物具有较好的荧光性能 ${ }^{[41]}$. 类 似地，如果以 TMS 保护的二炔化合物 33(图 3)合成对称 的双三唑，由于 33 中的稠合芳杂环本身具有很好的光 物理性能，而且形成双三唑产物后三唑基团能进一步扩 大共轭体系、提高荧光量子效率，故产物良好的荧光性 能使其可以应用于多种金属离子的检测 $[29,42,43]$. 例如, Bunz 等 ${ }^{[44]}$ 以炔 33 为原料，原位去保护后与含多个醚键 的叠氮化物 34(图 3)反应，可得到水溶性对称双三唑化 合物，其能检测水溶液中 $\mathrm{Cu}^{2+}$ 和 $\mathrm{Ni}^{2+}$.

如果叠氮化物 34(图 3)与含有两个 $N, N$-二炔丙基苯 胺的氟硼苂光染料 35(图 4)进行 Click 反应, 所得的高溶

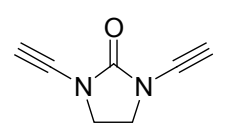

26

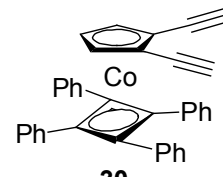

30<smiles>C#Cc1cccc(C#C)c1</smiles>

27

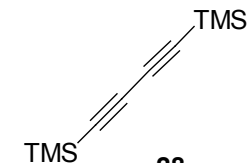

28

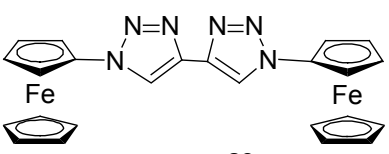

29

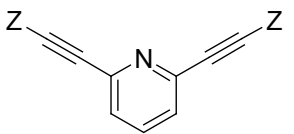

31, $\mathrm{Z}=\mathrm{H}, 32, \mathrm{Z}=\mathrm{TMS}$

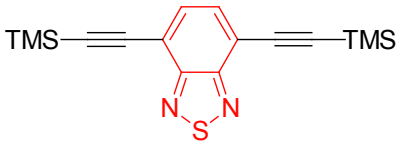

33
$\mathrm{N}_{3}+\mathrm{C}_{3}$

34

图 3 由二炔化物合成对称双三唑合成中的一些原料和产物

Figure 3 Some dialkynes and products in the symmetric synthesis of bis-1,2,3-triazoles 
解度产物含有四个三唑基团, 而且由于底物本身是氟砋 菼光染料, 使产物一方面有较高的苂光量子产率，另一 方面在近红外区有较强的吸收, 甚至该化合物高选择性 地对 $\mathrm{Cu}^{2+}$ 和 $\mathrm{Hg}^{2+}$ 进行络合时可以用肉眼观察到颜色的 变化 ${ }^{[45]}$. 最近, Achilefu 课题组 ${ }^{[46]}$ 也以氟硼苂光染料类 的二炔化物为原料，合成了对称的双三唑化合物，其有 较高的光稳定性和苂光量子效率, 在极性溶剂如 DMSO 中呈现出较好的溶解性, 可应用于生物成像研究.

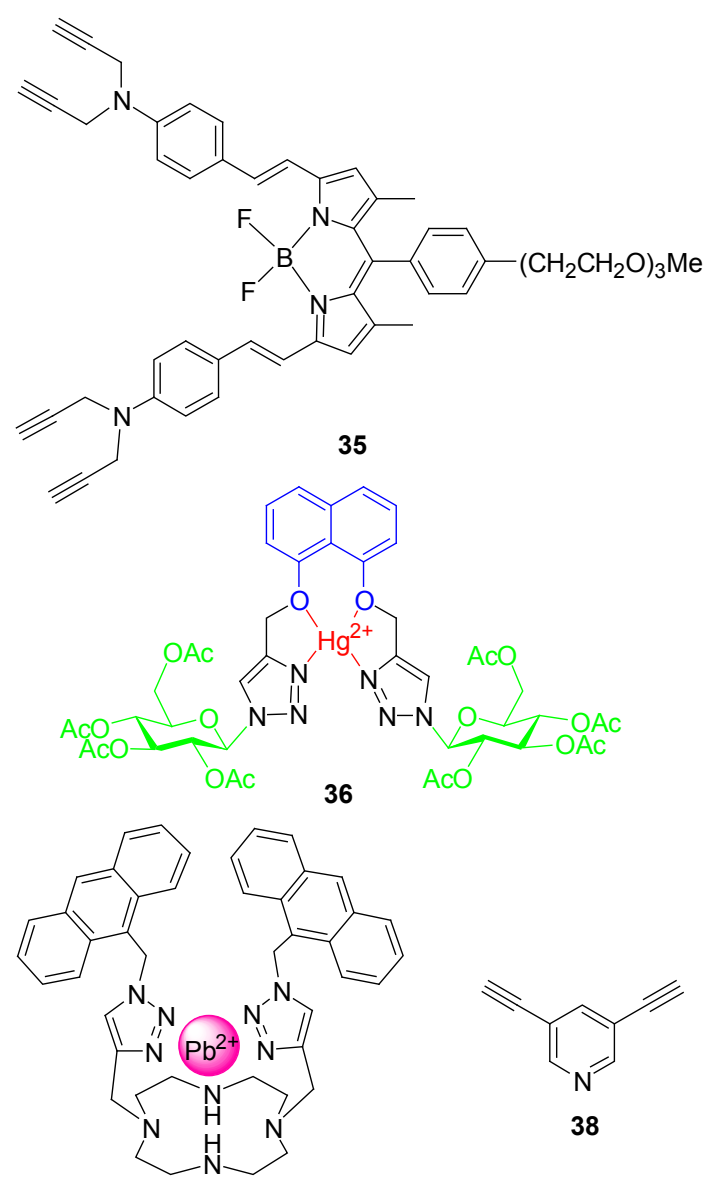

37

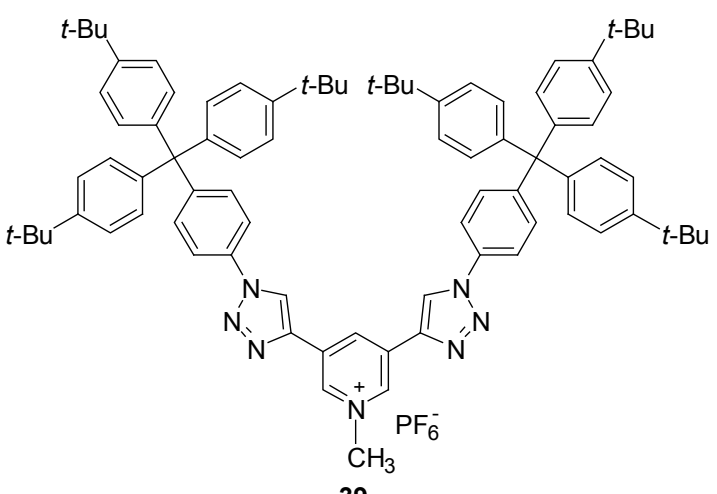

39

图 4 离子检测类对称双三唑合成中涉及的一些原料和目标 分子结构式

Figure 4 Some starting materials and products in the synthesis of symmetric bis-1,2,3-triazoles used as ion sensors
由于糖基不仅可提供较多的金属离子结合位点 ${ }^{[28]}$, 而且有利于改善三唑类化合物的亲水性 ${ }^{[47]}$ ，因此糖基 化与三唑官能团结合是利用化学传感器进行离子识别 研究中的一个新趋势 ${ }^{[28,48]}$. 例如, 最近 Das 课题组 ${ }^{[49]}$ 进 行了这方面的研究，菼光和凝胶实验表明，它们都能识 别 $\mathrm{Hg}^{2+}$. 后者的作用机制是, 当 $\mathrm{Hg}^{2+}$ 和对称双三唑的 配位时(化合物 36, 图 4), 其合适的空穴络合作用, 使有 机凝胶因子(如荟环、三唑基的 $\pi-\pi$ 堆积等)无法发挥作 用.

大环多胺中的多个氮原子, 一方面可以改善离子检 测受体水溶性, 另一方面也可参与配位. 因此, 大环多 胺类二炔化物与菼光性的蒽基叠氮化物进行反应，也能 获得具有很好检测效果的菼光探针. 例如, 最近余孝其 课题组 ${ }^{[50]}$ 首次得到了等当量的 $\mathrm{Pb}^{2+}$ 苂光传感器(化合物 37, 图 4), 其不仅检测灵敏度高, 不受其他金属离子影 响, 还可以进一步应用于活体细胞和胎牛血清中 $\mathrm{Pb}^{2+}$ 的 检测.

各种二炔化合物合成的对称双三唑类化合物，也可 用于阴离子的检测. 最近, Beer 课题组 ${ }^{[51]}$ 以含吡啶环的 二炔化物 38 为原料，经 Click 反应、甲基化等多步反应， 合成了对称双三唑化合物 39(图 4), 39 再与冠醚类化合 物作用, 可组装成轮烷一这种组合的超分子器件能识 别卤离子, 特别是溴离子.

双三唑类化合物不仅可以作为化学传感器应用于 离子检测，也可通过把三唑官能团作为亲水基与疏水基 间的连接模块而应用于新型的双子表面活性剂. 例如, Mohammed 等 ${ }^{[52]}$ 以廉价易得的 $D$-甘露醇为原料, 先合 成其二炔化物 40, 再与叠氮化物发生 Click 反应, 最终 可得到中心是以 $D$-甘露醇基元为亲水基的双三唑类表 面活性剂 41(图 5).

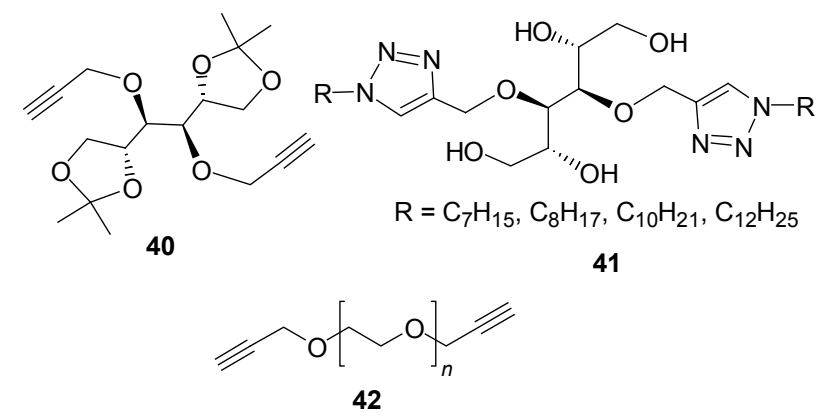

图 5 表面活性剂类对称双三唑合成中涉及的一些原料和目 标分子结构式

Figure 5 Some starting materials and products in the synthesis of symmetric bis-1,2,3-triazoles used as surfactants

一些含多醚链的二炔化合物(如 42, 图 5), 也是构 建双三唑类双子表面活性剂中亲水基的常见合成砌 块 ${ }^{[53]}$. 不仅如此, 这些亲水性的醚链也可作为含糖基药 
物设计中的连接模块 ${ }^{[54]}$. 当然, 直接以聚乙二醇 [HO$\left.\left(\mathrm{CH}_{2} \mathrm{CH}_{2} \mathrm{O}\right)_{n} \mathrm{H}, \mathrm{PEG}\right]$ 这种绿色溶剂 ${ }^{[55]}$ 的特殊功能进行双 三唑类药物的绿色合成, 也是可行的. 例如, Perumal 等 ${ }^{[56]}$ 在 PEG-400 反应体系中利用一锅法合成了一系列 具有抗菌活性的对称双三唑化合物.

利用各种二炔合成对称双三唑药物, 近年来也是关 注热点. 例如, Kaushik 等 ${ }^{[57]}$ 发现一些酯基连接的双三唑 (如化合物 43, 图 6)有较好的抗菌活性; Agarwal 课题 组 ${ }^{[58]}$ 则合成了同时含有苯并噻唑、卤素等官能团的对称 双三唑(如化合物 44), 它们具有较好的抗菌、抗真菌等 生物活性. 利用特殊的含磷二炔进行 Click 反应, 制备 的双三唑(如化合物 45) 可望应用于二膦酸酯类药物, 而 且此类药物分子(如化合物 46) 亦可通过含磷单炔与叠氮 化钠、二甾代烃一锅法合成 ${ }^{[59]}$.

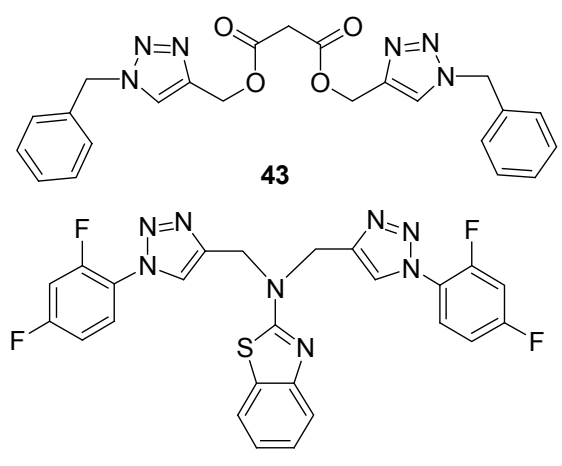

44
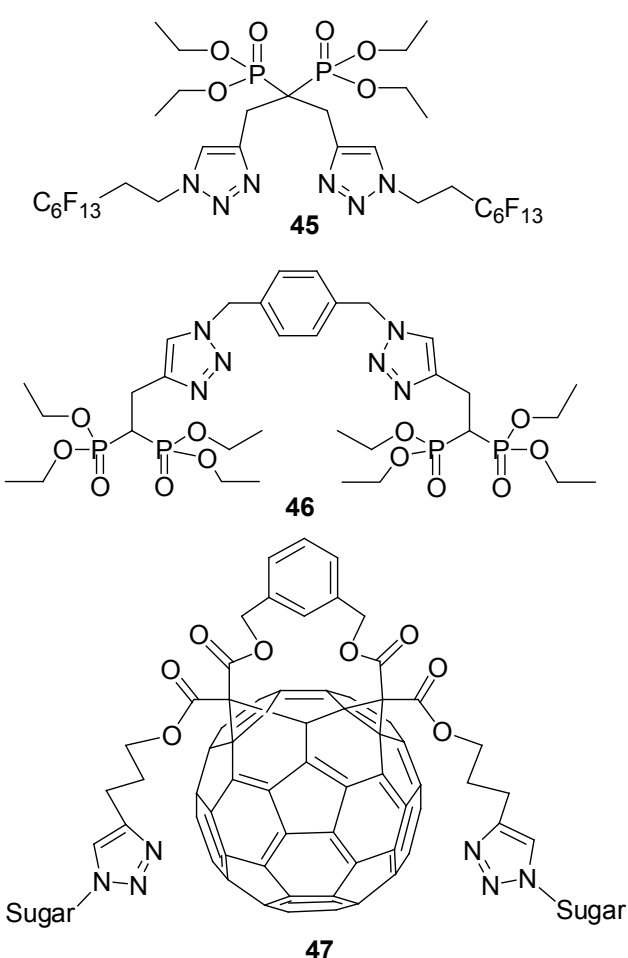

图 6 具有生物功能的对称双三唑目标分子

Figure 6 Some symmetric bis-1,2,3-triazole targeted molecules with biological functions
化学修饰的富勒烯在纳米材料科学与医药化学中 有广阔的应用前景，特别是富勒烯的糖缀合物具有类似 外源凝集素的生物活性，并且能够参与细胞间的分子识 别. 因此, de Freitas 等 ${ }^{[60]}$ 通过 Click 反应将糖基引入到富 勒烯衍生物中，以 $80 \% \sim 98 \%$ 的产率合成了系列新型富 勒烯的糖缀合物(如对称三唑化合物 47, 图 6).

\section{4 基于二炔化物与二叠氮化物的合成}

通过二炔化物和二叠氮化物的双 Click 反应，在控 制反应物低浓度的条件下也可有效地得到对称的双三 唑，并且通常是以三唑环为 “连接单元” 的中环或大环 化合物. 例如, Raghunathan 等 ${ }^{[61]}$ 以带有芴基的二炔(如 化合物 48, Eq. 8)与二叠氮化合物进行分子间双 Click 反 应，以 $60 \% \sim 70 \%$ 的产率合成了系列对称的多环双三唑 化合物。
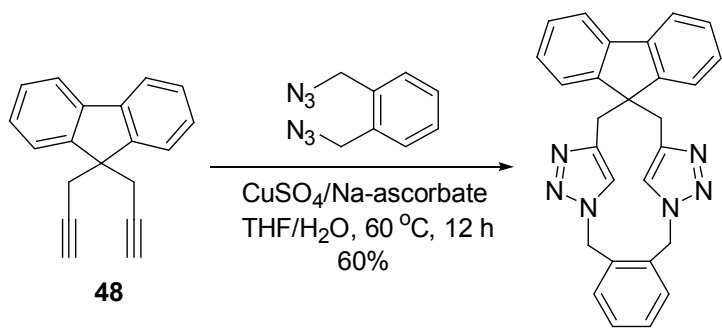

(8)

如果以多梄键的二炔化合物 49 与二叠氮化物 50 进 行双 Click 反应，在配体 $\mathbf{5 1}$ 存在下，冠醚类大环化合物 52 的产率可达 $65 \% \sim 70 \%$. 如果 49 在 $\left[\mathrm{Cu}\left(\mathrm{CH}_{3} \mathrm{CN}\right)_{4}\right]-$ $\left[\mathrm{PF}_{6}\right]$ 作用下先发生自组装形成配合物 $\mathbf{5 3}$, 再进行上述 的双 Click 反应关环，则能以 $80 \% \sim 92 \%$ 的高产率得到 索烃 54 (Scheme 4). 这种合成方法可为超分子化学与新 材料合成提供了新途径 ${ }^{[62]}$.

三唑环作为 “连接单元” 不仅有利于超分子器件的 合成，而且也可利用其带来的稳定性，构建具有多金属 核的大环配合物. 例如, Korybut-Daszkiewicz 课题组 ${ }^{[63]}$ 从金属配合物 55 出发, 在最后一步中以 $\mathrm{Cu}^{+}$和三 [(1-芐 基-1H-1,2,3-三唑-4-基)甲基]胺(TBTA)为催化剂，通过 分子间的双 Click 反应合成了系列新型的同核或异核配 合物 56 (Scheme 5). 这些巧妙设计得到的配合物作为杂 化的有机一无机电荷转移材料, 可望应用于电子开关器 件、数据储存系统等方面.

如果同一个底物中同时含有二末端炔和二叠氮基 结构，则可实现由分子间双 Click 反应到分子内双 Click 反应的转变, 从而构建包括三唑环在内的四环化合物. 例如, Mekni 等 ${ }^{[64]}$ 以化合物 $\mathbf{5 7}$ 为原料, 经多步反应得到 的底物 58 很易于进行分子内双 Click 反应, 甚至无 $\mathrm{Cu}^{+}$ 催化时产率更高, 得到对称双三唑化合物 59 具有抗菌 活性(Scheme 6). 


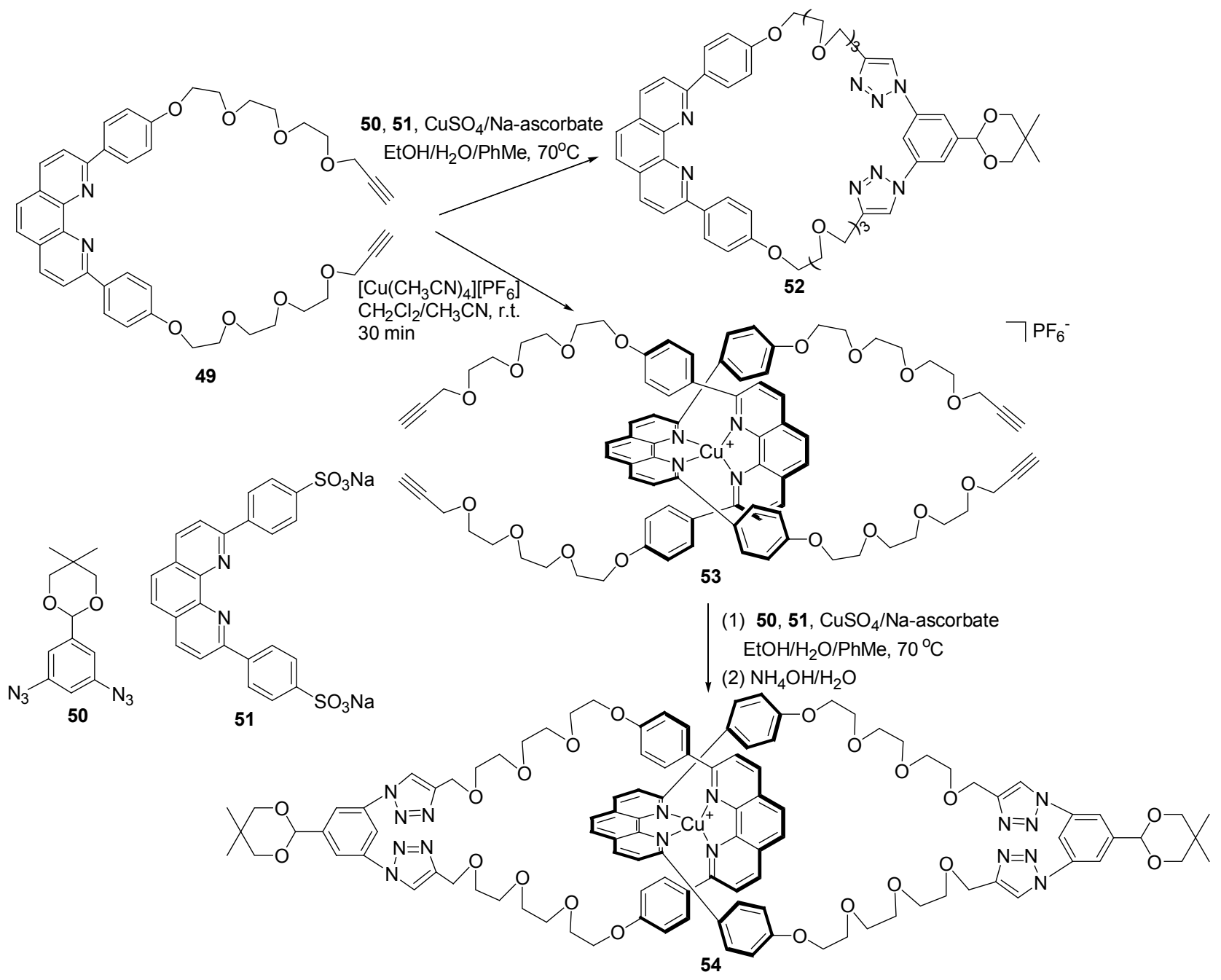

Scheme 4

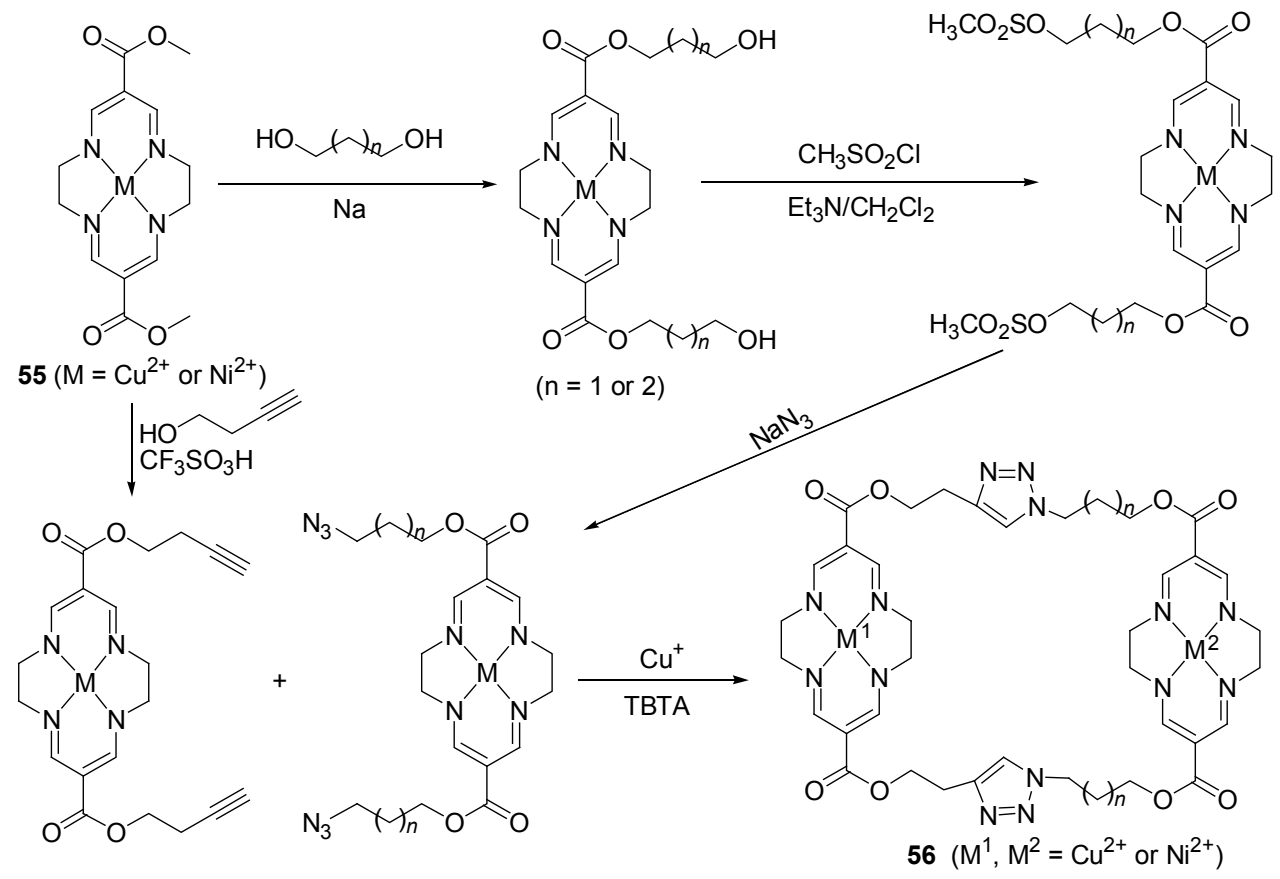

Scheme 5 

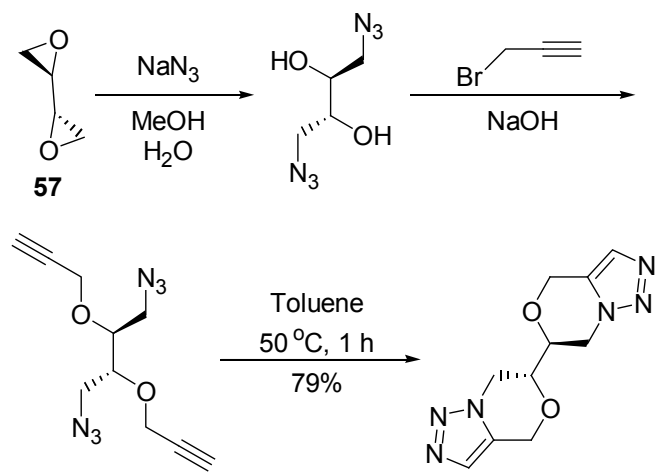

59

Scheme 6<smiles>C#CCC(CO)(CO)CC#CCCC</smiles>

60

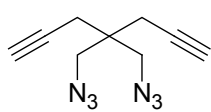

61
MW, $170{ }^{\circ} \mathrm{C}$<smiles></smiles>

Scheme 7

当二末端炔与二叠氮基结构巧妙地连接在同一个 季碳原子上时, 可以利用分子内的双 Click 反应得到具 有四环结构的螺环类双三唑. 例如, Sasai 等 ${ }^{[65]}$ 以二炔丙 基取代的丙二醇 60 为原料, 先多步反应得到底物 61 , 再在微波辅助的作用下发生分子内的双 Click 反应 (Scheme 7), 合成的双三唑化合物 62 经过烷基化后可制 备克量级的手性离子液体.

\section{2 不对称双-1,2,3-三唑化合物的合成}

近年来, 一些不对称双三唑化合物显示出比对称双 三唑更显著的生物活性 ${ }^{[2]}$, 引起了人们对不对称双三唑 合成的重视，但目前这方面的报道相对偏少.

\section{1 基于不同环境结构的炔基合成法}

对于一些非对称的二炔化合物, 由于本身端基炔带 来的环境结构差异, 在利用过量的叠氮化物进行双 Click 反应时, 很容易得到不对称的双三唑. 例如, Walsh 课题组 ${ }^{[66]}$ 通过这种双 Click 反应在肽类上引入糖 基，制备了糖基化的环状肽类抗生素; Engels 课题组 ${ }^{[67]}$ 以二炔化物 63(图 7)为原料, 在微波辅助和无溶剂条件 下, 以 $91 \%$ 的产率得到了不对称双三唑, 最终产物具有 抗菌和抗病毒的生物活性.

周成合课题组 ${ }^{[47]}$ 为了能增加目标化合物的水溶性, 将葡萄糖单元引入到不对称双三唑 64 中(图 7), 抗菌活 性测试表明, 64 中烷基链长对抗菌活性有显著的影响; 而本课题组 ${ }^{[68]}$ 则以氨基酸结构单元为中心, 利用非对<smiles>C#CCn1ncc(=O)n(CC#C)c1=O</smiles>

63

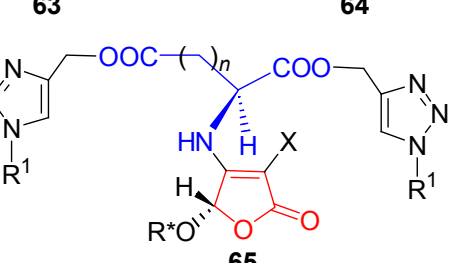

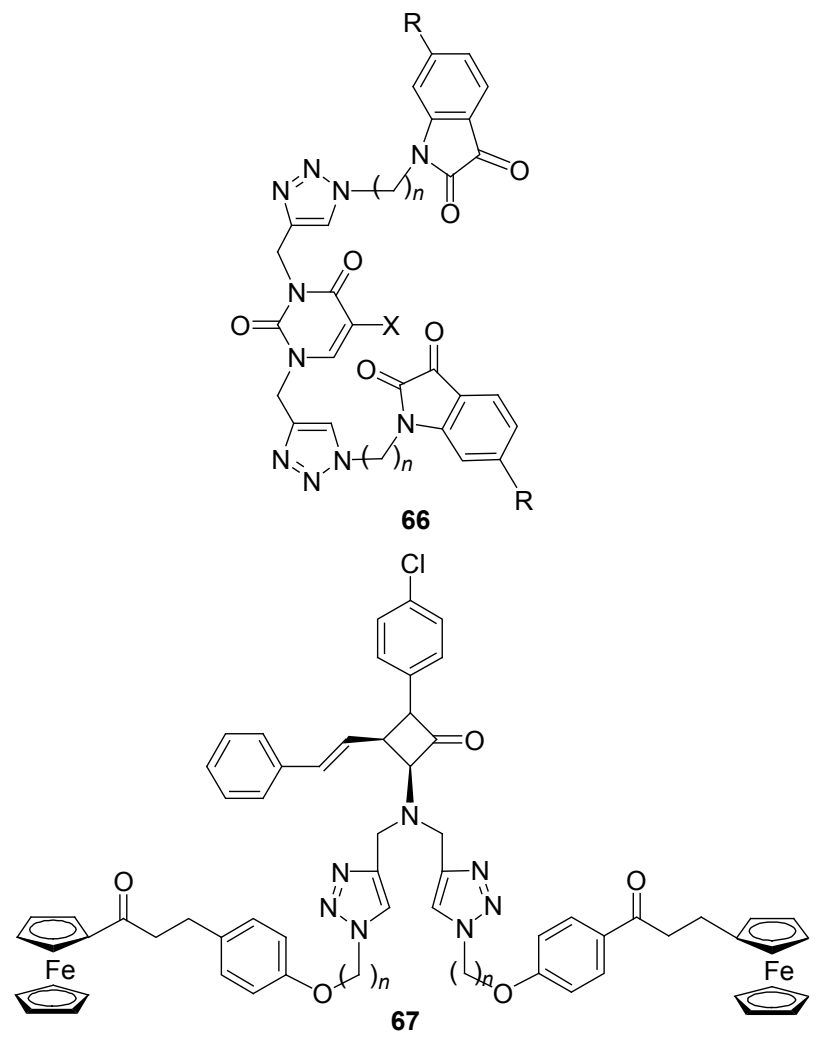

图 7 医药类不对称双三唑合成中涉及的一些原料和目标分 子结构式

Figure 7 Some starting materials and products in the synthesis of unsymmetrical bis-1,2,3-triazoles used as drugs

称二炔的双 Click 反应, 将具有生物活性的 $2(5 H)$-呋喃 酮模块 ${ }^{[69 ~ 71]}$ 与三唑模块 ${ }^{[72,73]}$ 组合在一起, 合成了系列 潜在的不对称双三唑药物(化合物 65, 图 7).

除抗菌、抗真菌等活性外，不对称双三唑还有其他 生物活性. 例如, Kumar 课题组 ${ }^{[74]}$ 以非对称二炔化物为 原料合成了双三唑化合物 66(图 7), 其对子宫颈癌、乳 腺癌、前列腺癌等癌细胞都具有抗癌活性. 最近, 他 们 ${ }^{[75]}$ 又利用非对称二炔的双 Click 反应, 开发了具有较 高的抗肺癌活性的不对称双三唑 67(图 7).

不考虑二炔底物中端基炔活性，直接利用过量叠氮 
化物合成的不对称双三唑, 也可应用于荧光传感器. 例 如, 汤杰课题组 ${ }^{[29]}$ 以荧光黄衍生的非对称二炔与氨基 酸基叠氮化物发生双 Click 反应，先得到不对称双三唑， 再脱去氨基的保护后, 最终得到的产物 68(图 8)可作为 苂光传感器或应用于标记生物分子.

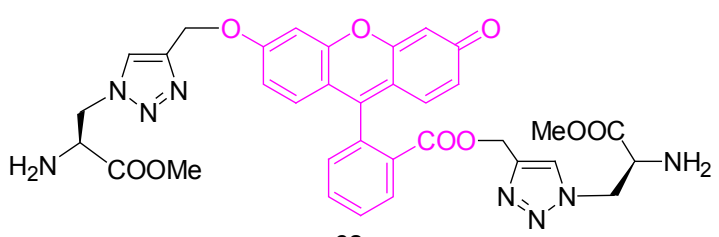

68

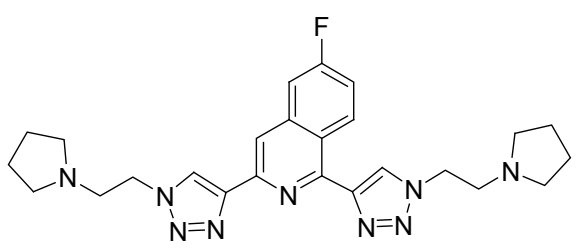

69

Figure 8 荧光传感器类不对称双三唑目标分子结构式

Figure 8 Some unsymmetrical bis-1,2,3-triazoles used as fluorescent sensors

最近, Dash 课题组 ${ }^{[76]}$ 以带有吡啶环的二炔化物为原 料, 利用双 Click 反应设计合成一种新型苂光分子探针 69(图 8). 这种水溶性化学传感器对于重金属和过渡金 属离子比较敏感. 当水溶液 $\mathrm{pH}$ 值为 7.0 时, 它对 $\mathrm{Zn}^{2+}$ 显示出苂光增强和高选择性优于其他生物相关的金属 离子. 不仅如此, 它还具有细胞膜渗透性, 可适用于细 胞内 $\mathrm{Zn}^{2+}$ 成像.

如果所用二炔化物原料分子中两个炔基的活性存 在明显的差异, 通过控制反应条件, 可使两个炔基依次 与不同的叠氮化物发生 Click 反应. 例如, Girard 等 ${ }^{[77]}$ 发 现，二炔化物 70 中靠近羰基的炔基具有更高的反应活 性, 可在无溶剂和催化剂的条件下首先与苠基叠氮化物 发生 Click 反应, 生成单三唑化合物 71, 其接着再与另 外的叠氮化物在大孔树脂 $\mathrm{A} 21$ 负载 $\mathrm{CuI}$ 的催化下进行第 二次 Click 反应，最终能生成不对称双三唑化合物 72 (Scheme 8).

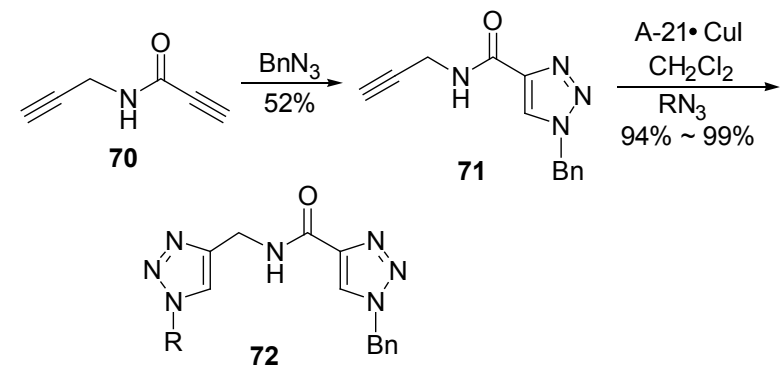

Scheme 8
进一步研究表明，第一步 Click 反应中的底物并不 仅仅局限于化合物 $\mathbf{7 0}$ 与茮基叠氮化物，一些类似物也 可, 甚至反应也能在丙酮中进行 ${ }^{[78]}$. 很明显, 利用这种 无保护底物的逐步双 Click 反应，可设计合成出组合性 更强的不对称双三唑化合物，从而更加有利于药物活性 的篎选. 最近, Girard 课题组 ${ }^{[79]}$ 对这样获得的系列不对 称双三唑进行了细胞毒性测试, 发现一些化合物具有抗 癌活性.

以环张力促进叠氮环加成反应 $(\mathrm{SPAAC})$ 合成三唑 时不需要额外的试剂, 其在活细胞蛋白质修饰、寡核苷 酸标记等生物化学领域已得到广泛应用 ${ }^{[80,81]}$, 特别是 SPAAC 与 CuAAC 联用更有利于三唑化合物在各种化 学生物学中的应用 ${ }^{[19,82,83]}$. 因此, Beal 等 ${ }^{[83]}$ 利用同时含 有环辛炔结构和末端炔结构的二炔化物为原料(如化合 物 73), 先进行无催化剂的 SPAAC 反应, 再与另一种叠 氮化物发生 CuAAC 反应，合成了可引入全氟烃基、糖 基和苂光基等多种特殊官能团的不对称双三唑化合物 74 (Scheme 9). 这种简单、高效、迅速而且无纯化过程 的一锅法模板反应，非常有利于开发以化学法增强的生 物治疗药物.
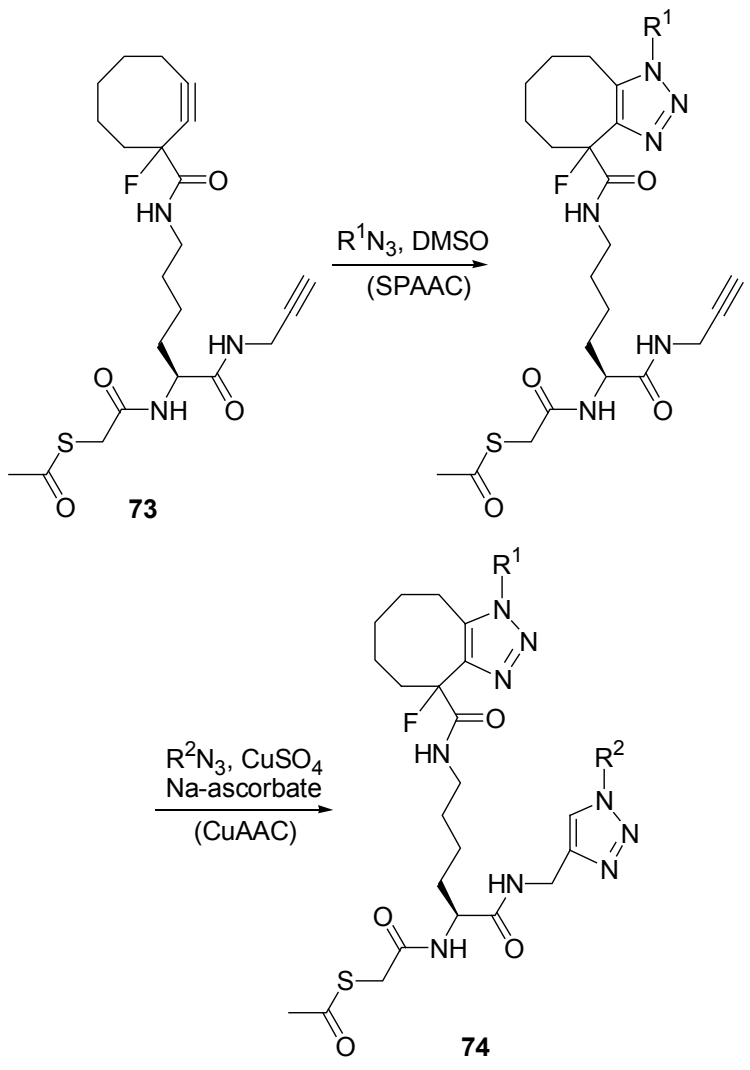

Scheme 9

\section{2 基于炔基保护的合成法}

虽然不同环境结构的二炔在活性不同时，可以连续 地、选择性地合成不对称双三唑化合物. 但遗憾的是, 
这种活性差异明显的二炔化合物并不多见，或制备方法 复杂. 因此, 目前基于炔基保护的不对称双三唑合成法 仍然是主流, 其基本的流程是：先保护其中的一个炔基， 使未保护的端基炔先发生 Click 反应，再脱保护后使另 一个炔基与新的叠氮化物发生第二次的 Click 反应.

例如, Aucagne 等 ${ }^{[84]}$ 以 TMS 保护的含肽链二炔化物 75 为原料, 没有保护基的末端炔烃优先与叠氮化物反 应, 得到单三唑化合物, 然后另一部分带有 TMS 保护基 的二炔化物继续与另一种叠氮化物, 通过 $\mathrm{AgBF}_{4}$ 脱保 护，并与新的叠氮化合物进一步发生 Click 反应，最后 可得到不对称双三唑化合物 76 (Scheme 10). 其中, 脱 保护与铜催化可采用一锅法进行. 这种方法为构建复杂 的肽类衍生物提供了一种有效的组合化学途径.

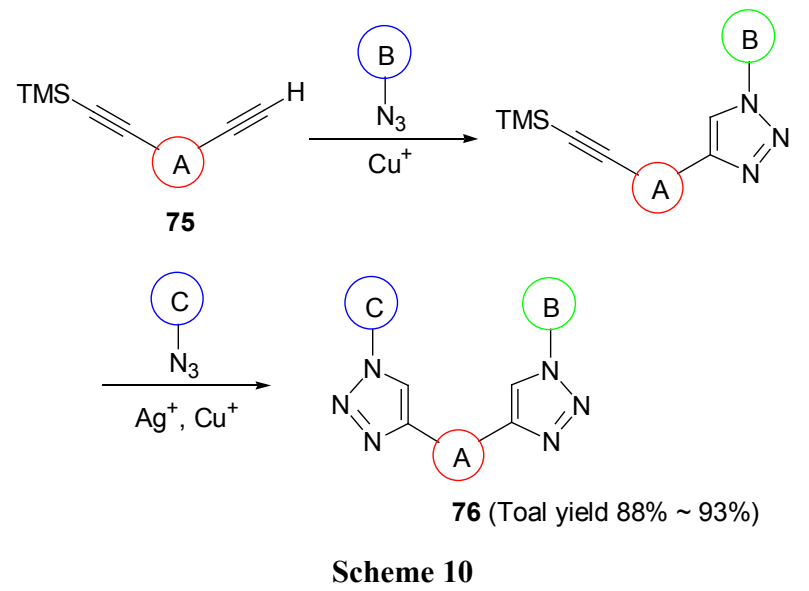

在炔基保护法合成不对称双三唑的报道中，带有 TMS 保护基的 1,3-丁二炔是一种常见的原料. 例如, Fiandanese 等 ${ }^{[85]}$ 以 1-TMS-1,3-丁二炔(77)作为原料, 先 得到单三唑, 再在四丁基氟化铵(TBAF)作用下脱保护, 并原位通过 $\mathrm{CuI} /$ 胺类催化的 Click 反应得到双三唑化合 物 78, 这可为合成不对称的 4,4'-双三唑化合物提供新方 法(Scheme 11).
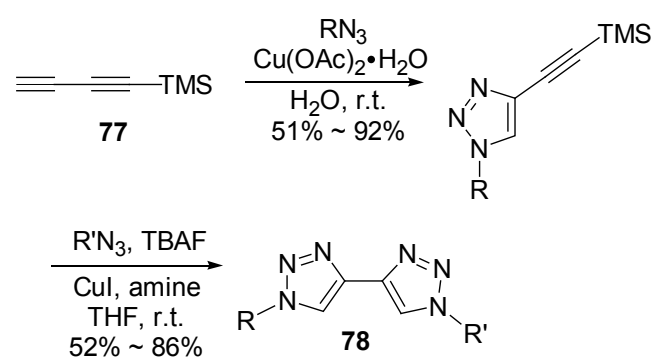

Scheme 11

此外，也可采用双 TMS 保护的 1,3-丁二炔(28)为底 物合成不对称双三唑. 例如, Aizpurua 等 ${ }^{[86]}$ 先使用脱保 护试剂 $\mathrm{MeLi} \cdot \mathrm{LiBr}$ 去掉一个保护基, 后来再用 $\mathrm{CsF}$ 去掉 第二个 TMS 保护基(Scheme 12). 这种简单、方便的合
成方法，可用于合成带有复杂官能团(如糖基、肽类等) 的双三唑化合物. 不足的是, 在合成单三唑 79 时, 硫酸 铜/抗坏血酸钠催化体系会引起 TMS 脱落而生成对称双 三唑副产物，故宜选择 CuI/DIPEA 催化体系.

如果使用相对于 TMS 保护基更加稳定的三异丙基 硅基(TIPS)作为保护基，不仅更容易控制反应的进行， 也可避免不同铜催化剂体系带来的烦恼. 例如, Simpson 等 ${ }^{[87]}$ 以 TIPS 保护的 1,3-丁二炔为原料, 利用 TBAF 脱去 TIPS 的保护, 采用一锅三步串联法合成了系列可用于 过渡金属配体的不对称双三唑化合物。
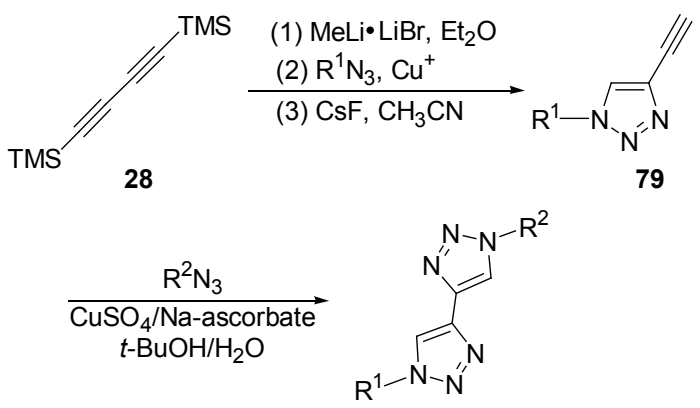

\section{Scheme 12}

\section{3 利用二叠氮化物合成不对称双三唑}

如果希望避免炔基保护带来的麻烦，也可以二叠氮 化物为原料合成不对称双三唑. 显而易见，如果所用二 叠氮化物是不对称的，即使炔原料是相同的，所得产物 一般都是不对称的. 例如, Benoist 等 ${ }^{[25]}$ 以二叠氮化物 80 为原料, 利用 Click 反应合成了不对称双三唑, 其水解 产物 81 能与铟、钎等过渡金属离子形成配合物而应用 于特定靶向的放射性药物(图 9).

类似地, Kamal 等 ${ }^{[88}$ 以不对称二叠氮化物发生的双 Click 反应，合成了可用于研究与 DNA 相互作用的化合 物 82(图 9). 最近, Turks 课题组 ${ }^{[89]}$ 以糖基修饰嘌呤衍生 物，获得的嘌呤类二叠氮化物(如化合物 83) 可合成系列 不对称双三唑化合物，这些产物都有良好的荧光性能， 甚至部分化合物的的苂光量子产率高达 $53 \%$; 而 Barros ${ }^{[90]}$ 和 Westermann 课题组 ${ }^{[91]}$ 均以糖基衍生的不对 称二叠氮化物合成了系列不对称双三唑，前者研究了它 们的微波合成技术，后者在合成基础进行了相关生物活 性研究.

如果不对称二叠氮化物中叠氮基的反应活性有明 显的差异，就能使其与不同的炔化合物依次进行 Click 反应，这有利于合成更多类型的不对称双三唑. 例如， Zhu 等 ${ }^{[92]}$ 发现，一些氮杂环叠氮化物(如化合物 84 87, 图 9)在发生 Click 反应时, 杂环上的氮原子参与对催化 剂的鳌合，使靠近该氮原子的叠氮基活性较高，能够优 先反应，而另一个叠氮基却只能在还原 $\mathrm{Cu}^{2+}$ 为 $\mathrm{Cu}^{+}$之后 
<smiles>CC(C)(C)OC(=O)NCC(N)CN</smiles>

80<smiles>NCC(Cn1cc(CN(CC(=O)O)CC(=O)O)nn1)n1cc(CN(CC(=O)O)CC(=O)O)nn1</smiles>

81<smiles>CC(=O)OCC1OC(n2cnc3c(N)cc(N)cc32)[C@H](OC(C)=O)[C@@H]1OC(C)=O</smiles>

83

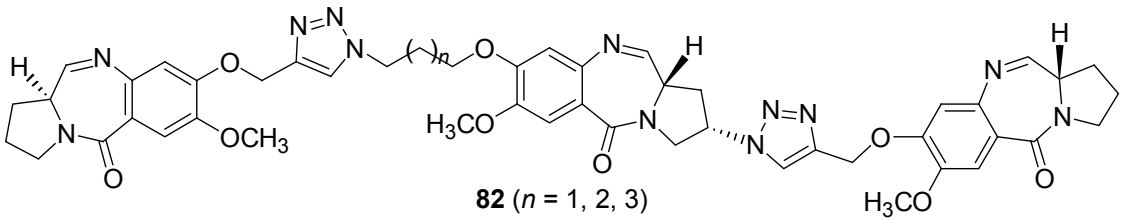<smiles>NCCCCOCc1cccc(CN)n1</smiles><smiles>N#CCCCCn1cc(CN)nn1</smiles><smiles>NCc1ccc2nc(CN)ccc2c1</smiles><smiles>NCc1ccc(CN)nc1</smiles>

图 9 不对称双三唑合成中不对称二叠氮化物及相关产物的结构式

Figure 9 Some diazides and products in the synthesis of unsymmetrical bis-1,2,3-triazoles

才进一步发生 Click 反应. 不仅如此, 反应中的三组分 能在中性水溶液中进行一锅法串联反应, 这可为不对称 双三唑在生物缀合领域的应用提供新路径.

对于对称的二叠氮化物, 如果希望与不同的炔组合 而合成不对称双三唑，使用保护手段是一种方式. 例如， Bundle 课题组 ${ }^{[93]}$ 以 1,3-二叠氮-2-丙醇为原料, 先经过两 步反应将一个叠氮基保护起来, 与炔化物发生第一次 Click 反应后再利用两步反应 “还原” 出叠氮基, 最后再 与另一种末端炔发生第二次 Click 反应, 最终可得有多 种组合方式的不对称双三唑, 但是其整体路线几长、总 产率低.

其实, 即使不使用不对称二叠氮化物, 而且对称二 叠氮化物原料是没有进行保护的, 但若在 Click 反应中 巧妙地控制反应物投料比, 并依次地采用不同的炔, 也 能得到不对称的双三唑. 最早采用该技术合成双三唑药
物的是 Overkleeft 课题组 ${ }^{[2]}$, 他们先采用单炔烃 $\mathbf{8 8}$ 与对 称的二叠氮化物 89 合成带有活性叠氮基的单三唑化合 物 90, 再与另一种含有炔基的药效基化合物 91 继续反 应, 得到的系列不对称双三唑 92 表现出了很好的生物 活性(Scheme 13).

最近，本课题组 ${ }^{[94]}$ 以 $N$-[5-烷氧基-2(5H)呋喃酮基] 氨基酸炔丙基酯(93)与 1,4-二叠氮丁烷为原料, 控制它 们的物质的量比为 $1: 2$, 成功地制得了活性叠氮基的 单三唑 94, 其再与各种炔化物进行室温 Click 反应, 即 可得不对称的双三唑(Scheme 14). 可见，这种基于对称 二叠氮化物制备不对称双三唑的合成方法，因原料易 得、简单易行而值得推广.

\section{4 利用特殊试剂合成法}

近年来，利用一些特殊试剂合成不对称双三唑也有<smiles>C#CCOc1ccc(C2(C)CC(C)(C)N(C(C)=O)c3ccc(NC(=O)c4ccc(-c5ccccc5)cc4)cc32)cc1</smiles>

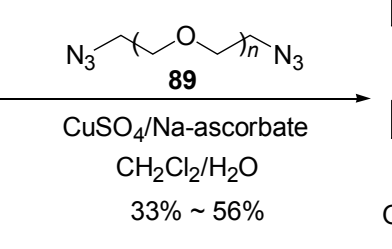

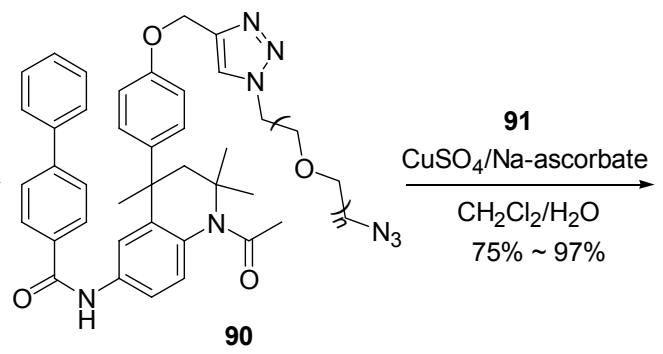

90<smiles>CSc1nc(-c2cccc(NCC(=O)NCc3cn(CCOCCn4cc(COc5ccc(C6(C)CC(C)(C)N(C(C)=O)c7ccc(NC(=O)c8ccc(-c9ccccc9)cc8)cc76)cc5)nn4)nn3)c2)c2c(N)c(C(=O)NC(C)(C)C)sc2n1</smiles><smiles>C#CCNC(=O)CNc1cccc(-c2nc(SC)nc3sc(C(=O)NC(C)(C)C)c(N)c23)c1</smiles>

Scheme 13 


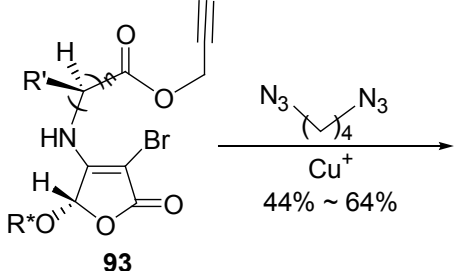

93<smiles>[Y9]Cn1cc(COC(=O)C([R])NC2=C(Br)C(=O)O[C@@H]2[R20])nn1</smiles>

94
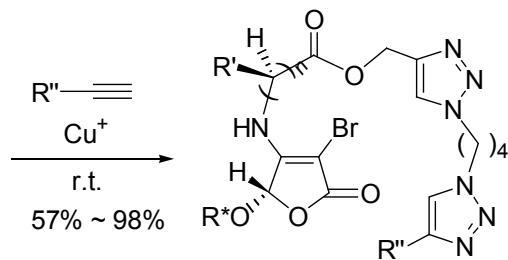

\section{Scheme 14}

报道. 例如, Kumar 课题组 ${ }^{[95,96]}$ 提出了一种利用格氏试 剂丙二烯基溴化镁 95 来合成不对称双三唑化合物的方 法, 其先是与叠氮化物发生串联反应得到 5-丁炔基三唑 96, 然后单三唑 96 再与另一种叠氮化物进行 Click 反应, 最终生成不对称的双三唑 97 (Scheme 15). 在两步反应 中均可引入糖基, 故可有效地控制目标化合物亲水性和 亲脂性两者间的平衡，从而有利于抗 HIV 药物的篮选.

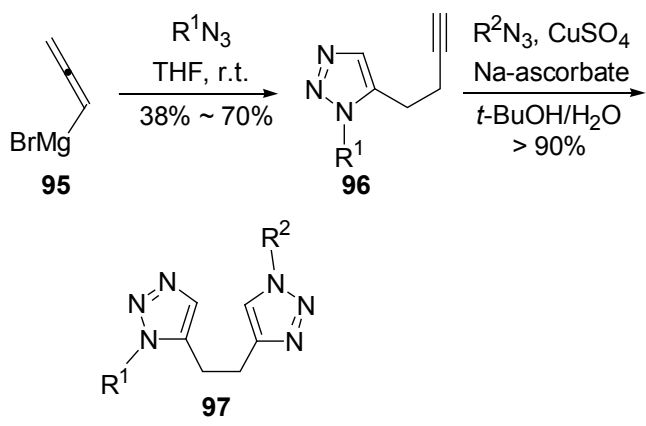

Scheme 15

Shi 课题组 ${ }^{[97]}$ 在苯并三氮唑的基础上, 先使其与炔 丙基醇反应，再进一步与叠氮化合物发生 Click 反应， 合成了系列不对称双三唑化合物(Scheme 16), 产物可 望应用为过渡金属配体.<smiles>[R]C#CC([R])n1nnc2ccccc21</smiles>

Scheme 16

\section{3 结束语}

总之，为了获得功能性更强的化合物或材料，目前 人们越来越关注于对称或不对称的双-1,2,3-三唑化合物 的合成, 特别是往往具有更佳效果的不对称双三唑. 事 实上，这种借助于 Click 反应，将具有不同结构、不同功 能基团的组分连接到一起, 以构建丰富多彩的化合物库
的合成研究, 已逐渐成为近年来的热点. 因此, 合成不 对称双三唑的方法学探索, 在未来的研究中仍须继续得 到足够的重视. 同时，针对目前一些目标分子合成中路 线繁琐等的不足，如何通过巧妙设计和高效合成，简洁 地得到目标化合物，从而拓展各种双-1,2,3-三唑化合物 (特别是功能性不对称双三唑)在药物合成、离子识别、 分子器件、化学生物学与表面活性剂合成等领域的应用, 也值得更进一步的研究.

\section{References}

[1] Meldal, M.; Tornoe, C. W. Chem. Rev. 2008, 108, 2952.

[2] Bonger, K. M.; Hoogendoorn, S.; van Koppen, C. J.; Timmers, C. M.; van der Marel, G. A.; Overkleeft, H. S. ACS Med. Chem. Lett. 2011, 2, 85 .

[3] Chan, T. R.; Hilgraf, R.; Sharpless, K. B.; Fokin, V. V. Org. Lett. 2004, 6, 2853.

[4] Wang, Q.; Chan, T. R.; Hilgraf, R.; Fokin, V. V.; Sharpless, K. B.; Finn, M. G. J. Am. Chem. Soc. 2003, 125, 3192.

[5] Service, R. F. Science 2008, 320, 868.

[6] Wu, P.; Feldman, A. K.; Nugent, A. K.; Hawker, C. J.; Scheel, A.; Voit, B.; Pyun, J.; Frechet, J. M. J.; Sharpless, K. B.; Fokin, V. V. Angew. Chem., Int. Ed. 2004, 43, 3928.

[7] Iha, R. K.; Wooley, K. L.; Nystrom, A. M.; Burke, D. J.; Kade, M. J.; Hawker, C. J. Chem. Rev. 2009, 109, 5620.

[8] Xiong, X.-Q.; Chen, H.-X. Chin. J. Org. Chem. 2013, 33, 1437 (in Chinese).

(熊兴泉，陈会新，有机化学, 2013, 33, 1437.)

[9] Sokolova, N. V.; Nenajdenko, V. G. RSC Adv. 2013, 3, 16212.

[10] Rostovtsev, V. V.; Green, L. G.; Fokin, V. V.; Sharpless, K. B. Angew. Chem., Int. Ed. 2002, 41, 2596.

[11] Angell, Y.; Burgess, K. Angew. Chem., Int. Ed. 2007, 46, 3649.

[12] Kwon, M.; Jang, Y.; Yoon, S.; Yang, D.; Jeon, H. B. Tetrahedron Lett. 2012, 53, 1606.

[13] Gonzalez, J.; Perez, V. M.; Jimenez, D. O.; Lopez-Valdez G.; Corona, D.; Cuevas-Yanez, E. Tetrahedron Lett. 2011, 52, 3514.

[14] Goyard, D.; Praly, J.-P.; Vidal, S. Carbohydr. Res. 2012, 362, 79.

[15] Jurček, O.; Cametti, M.; Pontini, M.; Kolehmainena, E.; Rissanen, K. Org. Biomol. Chem. 2013, 11, 4585.

[16] Lei, M.; Song, W.-Z.; Zhan, Z.-J.; Chen, X.-W.; Wang, Y.-G. Chin. J. Org. Chem. 2010, 30, 1854 (in Chinese). (雷鸣, 宋汪泽, 詹祖金, 陈小微, 王彦广, 有机化学, 2010, 30, 1854.)

[17] Wang, Z.-X.; Zhao, Z.-G. J. Heterocycl. Chem. 2007, 44, 89.

[18] Huang, Y.-E.; Gard, G.-L.; Shreeve, J.-M. Tetrahedron Lett. 2010, 51,6951 .

[19] Thirumurugan, P.; Matosiuk, D.; Jozwiak, K. Chem. Rev. 2013, $113,4905$.

[20] Oladeinde, O. A.; Hong, S. Y.; Holland, R. J.; Maciag, A. E.; 
Keefer, L. K.; Saavedra, J. E.; Nandurdikar, R. S. Org. Lett. 2010, 12,4256 .

[21] Gaur, M.; Goel, M.; Sridhar, L.; Ashok, T. D. S.; Prabhakar, S.; Dureja, P.; Raghunathan, P.; Eswaran, S. V. Monatsh. Chem. 2012, 143, 283.

[22] Huigens, R. W.; Rogers, S. A.; Steinhauer, A. T.; Melander, C. Org. Biomol. Chem. 2009, 7, 794.

[23] Karuturi, R.; Al-Horani, R. A.; Mehta, S. C.; Gailani, D.; Desai, U. R. J. Med. Chem. 2013, 56, 2415.

[24] Jurášeka, M.; Džubákb, P.; Sedlákc, D.; Dvořákováa, H.; Hajdúchb, M.; Bartůněkc, P.; Drašar, P. Steroids 2013, 78, 356.

[25] Camp, C.; Dorbes, S.; Picard, C.; Benoist, E. Tetrahedron Lett. 2008, 49, 1979.

[26] Hung, H.-C.; Cheng, C.-W.; Ho, I.-T.; Chung, W.-S. Tetrahedron Lett. 2009, 50, 302.

[27] Hung, H.-C.; Cheng, C.-W.; Wang, Y.-Y.; Chen, Y.-J.; Chung, W.-S. Eur. J. Org. Chem. 2009, 6360.

[28] Huang, H.-J.; Fang, H.-Y.; Chir, J.-L.; Wu, A.-T. Luminescence 2011, 26, 518.

[29] Li, C.; Tang, J.; Xie, J. Tetrahedron 2009, 65, 7935.

[30] Romero, T.; Orenes, R. A.; Tárraga, A.; Molina, P. Organometallics 2013, 32, 5740 .

[31] Wei, P.-F.; Yan, X.-Z.; Li, J.-Y.; Ma, Y.-J.; Yao, Y.; Huang, F.-H. Tetrahedron 2012, 68, 9179.

[32] Ol'shevskaya, V. A.; Makarenkov, A. V.; Kononova, E. G.; Petrovskii, P. V.; Verbitskiy, E. V.; Rusinov, G. L.; Charushin, V. N.; Hey-Hawkins, E.; Kalinin, V. N. Polyhedron 2012, 42, 302.

[33] Zhang, X. J.; Li, H. Y.; You, L. F.; Tang, Y.; Hsung, R. P. Adv. Synth. Catal. 2006, 348, 2437.

[34] Sarmiento-Sánchez, J. I.; Ochoa-Teran, A.; Rivero, I. A. ARKIVOC 2011, 177.

[35] Scott, S. O.; Gavey, E. L.; Lind, S. J.; Gordon, K. C.; Crowley, J. D. Dalton Trans. 2011, 40, 12117.

[36] Guisado-Barrios, G.; Bouffard, J.; Donnadieu, B.; Bertrand, G. Organometallics 2011, 30, 6017.

[37] Schweinfurth, D.; Büttner, N.; Hohloch, S.; Deibel, N.; Klein, J.; Sarkar, B. Organometallics 2013, 32, 5834.

[38] Singh, N.; Metla, B. P. R.; Elias, A. J. J. Organomet. Chem. 2012, $717,99$.

[39] Li, Y.-J.; Huffman, J. C.; Flood, A. H. Chem. Commun. 2007, 2692.

[40] Fletcher, J. T.; Bumgarner, B. J.; Engels, N. D.; Skoglund, D. A. Organometallics 2008, 27, 5430 .

[41] Mulla, K.; Shaik, H.; Thompson, D. W.; Zhao, Y. M. Org. Lett. 2013, 15, 4532.

[42] Li, C.; Henry, E.; Mani, N. K.; Tang, J.; Brochon, J. C.; Deprez, E.; Xie, J. Eur. J. Org. Chem. 2010, 2395.

[43] Ruan, Y.-B.; Yu, Y.-H.; Li, C.; Bogliotti, N.; Tang, J.; Xie, J. Tetrahedron 2013, 69, 4603.

[44] Brombosz, S. M.; Appleton, A. L.; Zappas, A. J.; Bunz, U. H. F. Chem. Commun. 2010, 46, 1419.

[45] Shi, W.-J.; Liu, J.-Y.; Ng, D. K. P. Chem. Asian J. 2012, 7, 196.

[46] Zhou, M.-Z.; Zhang, X.; Bai, M.-F.; Shen, D.-W.; Xu, B.-G.; Kao, J.; Ge, X.; Achilefu, X. RSC Adv. 2013, 3, 6756.

[47] Wei, J.-J.; Jin, L.; Wan, K.; Zhou, C.-H. Bull. Korean Chem. Soc. 2011, 32, 229.

[48] Lau, Y. H.; Rutledge, P. J.; Watkinson, M.; Todd, M. H. Chem. Soc. Rev. 2011, 40, 2848.

[49] Hemamalinia, A.; Das, T. M. New J. Chem. 2013, 37, 2419.

[50] Xu, H.-R.; Li, K.; Liu, Q.; Wu, T.-M.; Wang, M.-Q.; Hou, J.-T.; Huang, Z.; Xie, Y.-M.; Yu, X.-Q. Analyst 2013, 2329.

[51] White, N. G.; Beer, P. D. Org. Biomol. Chem. 2013, 11, 1326.

[52] Mohammed, A. I.; Abboud, Z. H.; Alghanimi, A. H. O. Tetrahe- dron Lett. 2012, 53, 5081.

[53] Mekni, N.; Baklouti, A. J. Soc. Chim. Tunisie 2009, 11, 15.

[54] Jervis, P. J.; Moulis, M.; Jukes, J. P.; Ghadbane, H.; Cox, L. R.; Cerundolo, V.; Besra, G. S. Carbohydr. Res. 2012, 356, 152.

[55] Bedard, A.-C.; Collins, S. K. J. Am. Chem. Soc. 2011, 133, 19976.

[56] Damodiran, M.; Muralidharan, D.; Perumal, P. T. Bioorg. Med. Chem. Lett. 2009, 19, 3611.

[57] Lal, K.; Kumar, A.; Pavan, M. S.; Kaushik, C. P. Bioorg. Med. Chem. Lett. 2012, 22, 4353.

[58] Singh, M. K.; Tilak, R.; Nath, G.; Awasthi, S. K.; Agarwal, A. Eur. J. Med. Chem. 2013, 63, 635.

[59] Skarpos, H.; Osipov, S. N.; Vorob'eva, D. V.; Odinets, I. L.; Lork, E.; Roeschenthaler, G.-V. Org. Biomol. Chem. 2007, 5, 2361.

[60] Pereira, G. R.; Santos, L. J.; Luduvico, I.; Alves, R. B.; de Freitas, R. P. Tetrahedron Lett. 2010, 51, 1022.

[61] Rajesh, R.; Periyasami, G.; Raghunathan, R. Tetrahedron Lett. 2010, 51, 1896.

[62] Megiatto, J. D.; Schuster, D. I. J. Am. Chem. Soc. 2008, 130, 12872.

[63] Mames, I.; Wawrzyniak, U. E.; Woźny, M.; Bilewicz, R.; Korybut-Daszkiewicz, B. Dalton Trans. 2013, 42, 2382.

[64] Mekni, N. H.; Baklouti, A. Heterocycles 2012, 85, 1727.

[65] Yoshida, Y.; Takizawa, S.; Sasai, H. Tetrahedron Lett. 2011, 52, 6877.

[66] Lin, H. N. Walsh, C. T. J. Am. Chem. Soc. 2004, 126, 13998.

[67] Krim, J.; Taourirte, M.; Engels, J. W. Molecules 2012, 17, 179.

[68] Huo, J.-P.; Lv, M.-X.; Wang, Z.-Y.; Li, Y.-Z. Chin. J. Chem. 2012, 30, 2411.

[69] Mao, C.-X.; Wang, Z.-Y.; Tan, Y.-H.; Xue, F.-L. Chin. J. Org. Chem. 2011, 31, 1377 (in Chinese).

(毛超旭, 汪朝阳, 谭越河, 薛福玲, 有机化学, 2011, 31, 1377.)

[70] Mo, Y.-Q.; Wang, Z.-Y.; Mei, W.-J.; Fu, J.-H.; Tan, Y.-H.; Luo, S.-H. Monatsh. Chem. 2012, 143, 443.

[71] Luo, S.-H.; Wang, Q.-F.; Wang, Z.-Y.; Peng P. Res. Chem. Intermed. 2013, 39, 2513.

[72] Tan, Y.-H.; Li, J.-X.; Xue, F.-L.; Qi. J.; Wang, Z.-Y. Tetrahedron 2012, 68, 2827.

[73] Huo, J. P.; Luo, J. C.; Wu, W.; Xiong, J. F.; Mo, G. Z.; Wang, Z. Y. Ind. Eng. Chem. Res. 2013, 52, 11850.

[74] Kumar, K.; Sagar, S.; Esau, L.; Kaur, M.; Kumar, V. Eur. J. Med. Chem. 2012, 58, 153.

[75] Kumar, K.; Carrere-Kremer, S.; Kremer, L.; Guerardel, Y.; Biot, C.; Kumar, V. Dalton Trans. 2013, 42, 1492.

[76] Midya, G. C.; Paladhi, S.; Bhowmik, S.; Saha, S.; Dash, J. Org. Biomol. Chem. 2013, 11, 3057.

[77] Elamari, H.; Jlalia, I.; Louet, C.; Herscovici, J.; Meganem, F.; Girard, C. Tetrahedron: Asymmetry 2010, 21, 1179.

[78] Elamari, H.; Meganem, F.; Herscovici, J.; Girard, C. Tetrahedron Lett. 2011, 52, 658.

[79] Elamari, H.; Slimi, R.; Chabot, G. G.; Quentin, L.; Scherman, D.; Girard, C. Eur. J. Med. Chem. 2013, 60, 360.

[80] Isaacman, M. J.; Corigliano, E. M.; Theogarajan, L. S. Biomacromolecules 2013, 14, 2996.

[81] Zhou, Q. H.; Gui, J. H.; Pan, C.-M.; Albone, E.; Cheng, X.; Suh, E. M.; Grasso, L.; Ishihara, Y.; Baran, P. S. J. Am. Chem. Soc. 2013, 135, 12994.

[82] Sapsford, K. E.; Algar, W. R.; Berti, L.; Gemmill, K. B.; Casey, B. J.; Oh, E.; Stewart, M. H.; Medintz, I. L. Chem. Rev. 2013, 113, 1904.

[83] Beal, D. M.; Albrow, V. E.; Burslem, G.; Hitchen, L.; Fernandes, C.; Lapthorn, C.; Roberts, L. R.; Selby, M. D.; Jones, L. H. Org. Biomol. Chem. 2012, 10, 548. 
[84] Aucagne, V.; Leigh, D. A. Org. Lett. 2006, 8, 4505.

[85] Fiandanese, V.; Bottalico, D.; Marchese, G.; Punzi, A.; Capuzzolo, F. Tetrahedron 2009, 65, 10573.

[86] Aizpurua, J. M.; Azcune, I.; Fratila, R. M.; Balentova, E.; Sagartzazu-Aizpurua, M.; Miranda, J. I. Org. Lett. 2010, 12, 1584.

[87] Doak, B. C.; Scanlon, M. J.; Simpson, J. S. Org. Lett. 2011, 13, 537.

[88] Kamal, A.; Shankaraiah, N.; Reddy. C. R.; Prabhakar, S.; Markandeya, N.; Srivastava, H. K.; Sastry, G. N. Tetrahedron 2010, 66, 5498.

[89] Kovalovs, A.; Novosjolova, I.; Bizdena, E.; Bizane, I.; Skardziute, L.; Kazlauskas, K.; Jursenas, S.; Turks, M. Tetrahedron Lett. 2013, 54, 850 .

[90] Potewar, T. M.; Petrova, K. T.; Barros, M. T. Carbohydr. Res. 2013, 79, 60 .

[91] Westermann, B.; Dörner, S.; Brauch, S.; Schaks, A.; Heinke, R.;
Stark, S.; van Delft, F. L.; van Berkel, S. S. Carbohydr. Res. 2013, 371,61 .

[92] Yuan, Z; Kuang, G.-C.; Clark, R. J.; Zhu, L. Org. Lett. 2012, 14, 2590.

[93] Guiard, J.; Fiege, B.; Kitov, P. I.; Peters, T.; Bundle, D. R. Chem. Eur. J. 2011, 17, 7438.

[94] Wang, Z.-Y.; Tan Y.-H.; Huo, J.-P.; Qi, J.; Li, D. In 12th International Symposium for Chinese Organic Chemists, Eds.: Zhai, H.-B.; Wang, W., Lanzhou University, Lanzhou, 2012, pp. 27.

[95] Banday, A. H.; Arora, B. S.; Alam, M. S.; Kumar, H. M. S. Helv. Chim. Acta 2007, 90, 2368

[96] Arora, B. S.; Shafi, S.; Singh, S.; Ismail, T.; Kumar, H. M. S. Carbohydr. Res. 2008, 343, 139.

[97] Yan, W.-M.; Wang, Q.-Y.; Chen, Y.-F.; Petersen, J. L.; Shi, X.-D. Org. Lett. 2010, 12, 3308. 